FERMILAB-PUB-06-289-T

ZH-TH 18/06

\title{
Collider searches and cosmology in the MSSM with heavy scalars
}

\author{
M. Carena ${ }^{1}$, A. Freitas ${ }^{2}$ \\ ${ }^{1}$ Fermi National Accelerator Laboratory, Batavia, IL 60510-0500, USA \\ ${ }^{2}$ Institut für Theoretische Physik, Universität Zürich, \\ Winterthurerstrasse 190, CH-8057 Zürich, Switzerland
}

\begin{abstract}
In a variety of supersymmetric extensions of the Standard Model, the scalar partners of the quarks and leptons are predicted to be very heavy and beyond the reach of next-generation colliders. For instance, the realization of electroweak baryogenesis in supersymmetry requires new sources of CP-violation, which can only be naturally accommodated with electric dipole moment constraints if the first and second generation scalar fermions are beyond the $\mathrm{TeV}$ scale. Also in focus-point supersymmetry and split supersymmetry the scalar fermions are very heavy. In this work, the phenomenology of scenarios with electroweak baryogenesis and in the focus point region at the LHC and ILC is studied, which becomes challenging due to the presence of heavy scalar fermions. Implications for the analysis of baryogenesis and dark matter are deduced. It is found that precision measurements of superpartner properties allow an accurate determination of the dark matter relic density in both scenarios, while important but only incomplete information about the baryogenesis mechanism can be obtained.
\end{abstract}




\section{Introduction}

Supersymmetry is one of the most compelling extensions of the Standard Model, with the possibility to explain the stabilization of the electroweak symmetry breaking scale, the existence of dark matter in the universe and the generation of the baryon-antibaryon-asymmetry at the electroweak phase transition (electroweak baryogenesis). The existence of dark matter in the universe has been firmly established by various experiments, and has been measured precisely by the Wilkinson Microwave Anisotropy Probe (WMAP) [1], in agreement with the Sloan Digital Sky Survey (SDSS) [2], $\Omega_{\mathrm{CDM}} h^{2}=0.1106_{-0.0075}^{+0.0056}$ at the $68 \%$ C.L. Here $\Omega_{\mathrm{CDM}}$ is the ratio of the dark matter energy density to the critical density $\rho_{\mathrm{c}}=3 H_{0}^{2} /\left(8 \pi G_{\mathrm{N}}\right)$, where $H_{0}=h \times 100 \mathrm{~km} / \mathrm{s} / \mathrm{Mpc}$ is the Hubble constant and $G_{\mathrm{N}}$ is Newton's constant. In the Minimal Supersymmetric extension of the Standard Model (MSSM), the stable lightest neutralino is an attractive candidate for dark matter.

The process of electroweak baryogenesis requires a sufficiently strongly first order electroweak phase transition, $v\left(T_{\mathrm{c}}\right) / T_{\mathrm{c}} \gtrsim 1$, where $v\left(T_{\mathrm{c}}\right)$ denotes the Higgs vacuum expectation value at the critical temperature $T_{\mathrm{c}}[3]$. Moreover, new sources of $\mathrm{CP}$ violation in addition to the CKM matrix phase are necessary [4]. Loop effects of light scalar top quarks (stops) can induce a strongly first order electroweak phase transition, thus generating the out-ofequilibrium condition for electroweak baryogenesis [5-8]. In addition, $\mathrm{CP}$ violation in the chargino sector of the MSSM can explain the magnitude of the baryon asymmetry.

The parameter space for successful electroweak baryogenesis in the MSSM is already highly constrained. The lightest stop mass needs to be in the range of roughly 115 to 140 $\mathrm{GeV}$, whereas the Higgs boson involved in the electroweak phase transition must be lighter than about $120 \mathrm{GeV}$ [5-9]. Furthermore, a CP-violating phase in the chargino sector is highly constrained by bounds on electric dipole moments $[10,11]$.

Most MSSM scenarios predict a dark matter density larger than the measured value. However, the requirement of a light stop from the baryogenesis mechanism suggests that coannihilation between the stop and the lightest neutralino can bring down the relic density to the proper region. It has been shown that the co-annihilation is efficient for mass differences between the light stop and the lightest neutralino, that are smaller than about $30 \mathrm{GeV}[12]$.

Light stops can be searched for at the Tevatron, with a reach up to stop masses of about $170 \mathrm{GeV}$ for $2-4 \mathrm{fb}^{-1}$ of integrated luminosity [13]. However, if the stop-neutralino mass difference is less than 30-50 GeV, the stop signal cannot be identified at the Tevatron, due to the reliance on a trigger for missing transverse energy. At the next generation of colliders, the situation looks more promising. The Large Hadron Collider (LHC), depending on the region of parameter space, can see a signal from stops in gluino cascades, while a future international $e^{+} e^{-}$linear collider (ILC) has excellent capabilities to discover and analyze light stops $[9,14-16]$.

In order to allow sufficiently large $\mathrm{CP}$ violating phases for baryogenesis in the chargino sector, but evade current experimental electric dipole moment bounds [17], without invoking miraculous cancellations, the sfermions of the first two generations need to be heavier than a few $\mathrm{TeV}$, thus effectively decoupling from collider experiments. This situation has grave consequences for the investigation of supersymmetry at the LHC and ILC. While the lightest 
Higgs boson, and possibly some of the heavier Higgs boson, are within experimental reach as in other MSSM scenarios, only few supersymmetric particles are likely to be kinematically accessible in this scenario: neutralinos and charginos, the light stop and potentially the gluino and some sbottom and stau states. Thus it becomes much more difficult to identify and measure the properties of these particles. A similar situation occurs in focus-point [18] and split supersymmetry scenarios [19], where all sfermions are predicted to be very heavy.

In this work, it is investigated how a scenario with heavy sleptons and squarks can be studied at the LHC and the ILC. The analysis is carried out in detail for an electroweak baryogenesis scenario and for a focus-point scenario. Split supersymmetry is not studied separately, but the conclusions are very similar to the focus-point case. After reviewing the definition of the relevant parameters as well as experimental and theoretical constraints in section 2, the phenomenology of the baryogenesis scenario is discussed in section 3. while section 4 is devoted to the focus-point scenario. The results are based on a phenomenological analysis, including backgrounds and systematic experimental limitations, but without a realistic detector simulation and based on tree-level formulae only. For both scenarios, the cosmological implications to be gained from collider results are derived for elucidating the nature of baryogenesis and dark matter. For the main part of the work, it is assumed that both electron and positron beam polarization are available at the ILC. In section 5 it is studied how the results change without positron polarization. Finally, the conclusions are given in section 6 .

\section{Baryogenesis and focus-point scenarios in the MSSM}

Baryogenesis in the MSSM requires an additional source of CP violation beyond the Standard Model CKM matrix. Within the MSSM, the dominant source are chargino and neutralino loops, with a contribution proportional to $\operatorname{Im}\left\{\mu M_{1,2}\right\}[20,21]$. Here $\mu$ is the Higgs/higgsino parameter and $M_{2}$ and $M_{1}$ are the soft $\mathrm{SU}(2)$ and $\mathrm{U}(1)$ gaugino parameters, respectively. To generate a sufficiently large baryon asymmetry, the charginos are required to be relatively light, $m_{\tilde{\chi}_{1}^{ \pm}} \sim \mathcal{O}$ (a few $\left.100 \mathrm{GeV}\right)$. In addition, the CP-violating phase needs to be sizable, $\arg \left(\mu M_{2}\right) \gtrsim 0.1[20]$.

A very large $\mathrm{CP}$-violating phase, on the other hand, is restricted by experimental bounds on the electric dipole moments of the electron, neutron and ${ }^{199} \mathrm{Hg}$ nucleus [17]. The leading contributions from one-loop sfermion-gaugino loops [10,11] become small for large masses of the first two generation sfermions of several $\mathrm{TeV}$ or for large cancellations between the sfermion mass and mixing parameters. Here, to avoid the constraints from the one-loop contributions, the sleptons and squarks of the first two generations are assumed to have masses of about $10 \mathrm{TeV}$. Then the leading contributions from supersymmetric particles to the electric dipole moments come from two-loop diagrams. For moderate values of $\tan \beta<10$, the ratio of the vacuum expectation values of the two Higgs doublets, and the CP-violating phase, it has been shown $[22,23]$ that the two-loop effects are in agreement with current experimental constraints.

Generically, at the LHC many supersymmetric particles could be observed in the decay 
chain of squarks, whereas at ILC selectron production would be one of the cleanest and most precise testing grounds for supersymmetry. However, in the baryogenesis motivated scenario, the large mass of the first and second generation sfermions effectively decouples them from observable processes. This has a large impact on the prospects for future collider experiments, since the squark and slepton production channels mentioned above are not available. Likewise, the dynamics of baryogenesis and dark matter annihilation are governed by the light particles in the MSSM spectrum, which include, beside the stops, the charginos and neutralinos. Therefore the following analysis focuses on the phenomenology of the chargino and neutralino particles.

The spectrum of the two charginos and four neutralinos is described by the parameters $\mu, M_{1}, M_{2}$ and $\tan \beta$. A CP-violating phase in $M_{2}$ can always be transferred into the $\mu$ parameter by means of a unitary transformation. In principle, there can also be non-trivial phases in the gaugino parameters $M_{1}$ and $M_{3}$. While the effect of a phase of $M_{3}$ on electroweak baryogenesis is small, a complex phase of $M_{1}$ could have interesting consequences, but is not investigated further here. Therefore in the following all gaugino soft parameters are assumed real, while the generation of the baryon asymmetry is connected with a phase in the $\mu$ parameter, $\mu=|\mu| \times e^{i \phi_{\mu}}$.

For definiteness, the specific MSSM parameter point BGEN will be considered in the following, as defined in the appendix. Since the allowed parameter space for baryogenesis in the MSSM is already highly constrained by experimental bounds, this particular scenario is typical for the general MSSM baryogenesis case.

In the MSSM Higgs sector, the tree-level masses of the CP-even neutral Higgs bosons $h^{0}$ and $H^{0}$ and the charged scalar $H^{ \pm}$can be expressed through the gauge boson masses, the mass of the pseudo-scalar Higgs boson, $m_{\mathrm{A}^{0}}$, and $\tan \beta$. The Born relations are however significantly modified by radiative corrections, with dominant effects originating from top and stop loops. The mass of the Higgs state related to electroweak symmetry breaking (in most cases $h^{0}$ ) is very sensitive to the stop spectrum. In order to be consistent with the bound $m_{\mathrm{h}^{0}} \gtrsim 114.4 \mathrm{GeV}$ from direct searches at LEP [24] and with one light stop state, the heavier stop mass has to be above about $1 \mathrm{TeV}$ and the trilinear coupling $A_{t}$ has to be sizable [7]. Constraints from electroweak precision data, in particular the $\rho$ parameter, are satisfied when the light stop is mainly right-chiral. This is naturally achieved for values of the stop supersymmetry breaking parameters $m_{\tilde{\mathrm{Q}}_{3}}^{2} \gtrsim 1 \mathrm{TeV}^{2}$ and $m_{\tilde{\mathrm{U}}_{3}}^{2} \lesssim 0$, respectively. The stop mixing parameter $X_{t}=\mu \cot \beta-A_{t}$ is bounded from below by the Higgs boson mass constraint from LEP and from above by the requirement of the strength of the first order electroweak phase transition, leaving the allowed range $0.3 \lesssim\left|X_{t}\right| / m_{\tilde{\mathrm{Q}}_{3}} \lesssim 0.5$ [7]. The value of $\tan \beta$ is also constrained to the range $5 \lesssim \tan \beta \lesssim 10$, with the upper bound stemming from present electric dipole moment limits and the lower bound is again related to the LEP Higgs mass limit. The latter can be weakened for large values of $m_{\tilde{Q}_{3}}$ of several TeV. Also values of $m_{\mathrm{A}^{0}}$ larger than about $200 \mathrm{GeV}$ are preferred in order to be compatible with the electric dipole moment bounds.

The MSSM Higgs masses with CP violation have been calculated including complete oneloop and leading two-loop corrections, see e.g. Ref. [25]. In this work, however, the process of baryogenesis at the electroweak phase transition is computed with the program of Refs. [20, 
22], which includes only one-loop corrections to the zero temperature Higgs potential. Since the allowed mass range for the Higgs boson is constrained by the mechanism of electroweak baryogenesis, for consistency the Higgs mass is determined by the minimization of the oneloop effective potential. This implies that only one-loop corrections are included in the calculation of the Higgs mass as well. Although when including two-loop corrections, the values of the fundamental parameters will change for given values of $m_{\mathrm{h}^{0}}$ and $v\left(T_{\mathrm{c}}\right) / T_{\mathrm{c}}$, the correlation between $m_{\mathrm{h}^{0}}$ and $v\left(T_{\mathrm{c}}\right) / T_{\mathrm{c}}$ is expected not to be strongly modified ${ }^{1}$.

Focus point supersymmetry [18] was suggested to solve the supersymmetric flavor and $\mathrm{CP}$-problems by raising the masses of all scalars to several $\mathrm{TeV}$. It was observed that for certain parameter combinations, the Higgs parameters have an infrared quasi-fixed point, thus making the weak scale soft parameter $m_{H_{u}}$ of one of the Higgs doublets highly insensitive to the value of the other scalar masses. In this way, naturalness is preserved even for very large sfermion masses.

Here, as a concrete example, the focus point scenario LCC2, as defined in the appendix, will be analyzed in detail. The collider phenomenology of this scenario has been studied previously in Ref. [27], and cosmological implications were discussed in Ref. [28]. In this report, the phenomenological discussion of the earlier works is extended, and the scenario compared to the baryogenesis case.

\section{$3 \quad$ Baryogenesis scenario}

The LHC experiments will be able to probe a light Higgs boson with Standard-Model-like couplings to the gauge bosons, as required by electroweak baryogenesis. The potential for discovery of light stops has been studied in detail for the LHC [14] and ILC $[9,15,16]$. If gluinos are not too heavy, the discovery of light stops in gluino decays via the process $p p \rightarrow \tilde{g} \tilde{g} \rightarrow t t \tilde{t}^{*} \tilde{t}^{*}, \overline{t t} \tilde{t} \tilde{t}$ has been shown to be effective for small stop-neutralino mass differences [14]. Stops can be discovered with more than five standard deviations through the decay of gluinos if the gluino mass is below $900 \mathrm{GeV}$, the stop-neutralino mass difference about $40 \mathrm{GeV}$, and all other squarks relatively heavy. It was also shown [14] that if the other squarks are not much heavier than $1 \mathrm{TeV}$, even in the stop-neutralino co-annihilation region with $m_{\tilde{t}_{1}}-m_{\tilde{\chi}_{1}^{0}} \lesssim 30 \mathrm{GeV}$, stop discovery is possible in the decay of those squarks. However, further studies are needed to establish whether the stop-neutralino co-annihilation region can be explored at the LHC for first and second generation squarks with masses of several $\mathrm{TeV}$. In this parameter region, the decay products of the stops are very soft and thus escape direct detection, so that only the final state products of the two top quarks from gluino decay are observable. Nevertheless, the signature of same-sign top quarks and large missing energy is likely sufficient for the discovery of a new physics signal, even if the stops are not directly identified. When discovered, the analysis of the kinematical decay distributions of the stops at the LHC can be used to extract information about the relationship between

\footnotetext{
${ }^{1}$ An analysis including two-loop corrections to the effective potential is in progress [26].
} 
stop, gluino and lightest neutralino masses [14], but an independent determination of the stop or neutralino mass seems difficult.

At the ILC, light stops can be discovered for stop-neutralino mass differences down to about $5 \mathrm{GeV}$, independent of other MSSM parameters [9]. In addition, precise measurements of the stop mass and mixing angle can be performed, with a typical stop mass error of about $1 \%$, assuming $80 \%$ beam polarization for electrons and $50 \%$ for positrons.

\subsection{Chargino and neutralino studies at the LHC}

In this kind of scenarios, the analysis of neutralinos and charginos is very difficult. Supersymmetric particles are mainly produced through decay cascades of gluino and squarks, which can have large production cross-sections at the LHC. If however all squarks except the light stop are rather heavy, only the stop will be produced at sizeable rates and it will dominate the decays of the gluinos as well. However, a stop in accordance with electroweak baryogenesis is so light that it typically can only decay into the lightest neutralino, so that no information about the other neutralinos and charginos can be gained from stop processes.

The only sfermion whose mass is not constrained by theoretical or experimental bounds in the MSSM baryogenesis scenario, is the right-chiral sbottom quark. The left-chiral sbottom is connected through $\mathrm{SU}(2)$ symmetry to the left-chiral stop, which needs to be very massive to satisfy the LEP Higgs bound [24]. The right-chiral sbottom, on the other hand, directly decays into a bino neutralino, with negligible branching ratios into other neutralinos or charginos. Note that the light sbottom state is expected to be almost completely right-chiral in this scenario, since large mixing between left- and right-chiral sbottom states is suppressed when the light mass eigenstate is supposed to be much lighter than the heavy state.

Finally, charginos and neutralinos can also be produced directly through Drell-Yan-type processes. However, direct production processes of neutralino pairs have too small rates compared to the backgrounds at the LHC [29]. On the other hand, the production of a light chargino with a neutralino can have sizeable rates, but in a baryogenesis scenario, the chargino will mainly decay into the light stop. In the sample scenario BGEN (see appendix), the chargino branching fraction into stops and bottom quarks is more than $99.9 \%$. As a result, the production of mixed neutralino-chargino pairs will lead to at most one lepton plus jets in the final state, instead of the typical tri-lepton signature for mSUGRA scenarios. Unfortunately, the one lepton plus jets signature is totally swamped by $W$-boson background.

Thus the only observable supersymmetric particles at the LHC are, depending on the region of parameter space, the gluino, the light stop, potentially the light sbottom, and the missing energy signature of the lightest neutralino.

\subsection{Chargino and neutralino studies at the ILC}

In the given scenario, stops, charginos and neutralinos can be studied precisely at a future linear collider. The prospective measurements for the stop are studied in detail in Ref. [9]. Here the chargino and neutralino phenomenology is investigated. The characteristic feature of the baryogenesis scenario is that while the charginos and neutralinos can be light, the first 


\begin{tabular}{|r||rrr|}
\hline$e^{+} e^{-} \rightarrow \tilde{\chi}_{i}^{0} \tilde{\chi}_{j}^{0}$ & $\tilde{\chi}_{i}^{0}=\tilde{\chi}_{2}^{0}$ & $\tilde{\chi}_{3}^{0}$ & $\tilde{\chi}_{4}^{0}$ \\
\hline \hline$\tilde{\chi}_{j}^{0}=\tilde{\chi}_{1}^{0}$ & 0.7 & 25.1 & 0.07 \\
$\tilde{\chi}_{2}^{0}$ & 0.4 & 62.0 & 0.005 \\
$\tilde{\chi}_{3}^{0}$ & & - & - \\
$\tilde{\chi}_{4}^{0}$ & & & - \\
\hline \hline$e^{+} e^{-} \rightarrow \tilde{\chi}_{i}^{ \pm} \tilde{\chi}_{j}^{\mp}$ & $\tilde{\chi}_{i}^{ \pm}=\tilde{\chi}_{1}^{ \pm}$ & $\tilde{\chi}_{2}^{ \pm}$ & \\
\hline \hline$\tilde{\chi}_{j}^{\mp}=\tilde{\chi}_{1}^{\mp}$ & 665 & 28 & \\
$\tilde{\chi}_{2}^{\mp}$ & & - & \\
\hline
\end{tabular}

Table 1: Tree-level production cross-sections in fb at $\sqrt{s}=500 \mathrm{GeV}$ with unpolarized beams for the reference point BGEN.

generation sleptons are heavy. A similar situation arises in focus point supersymmetry and has been studied in previous works $[27,30]$. Here the study of Ref. [30] is extended by including more observables, simulations for signal and background processes and by considering a $\mathrm{CP}$-violating phase in the system.

In general, future collider experiments will not provide enough independent measurements to extract all chargino and neutralino mass and mixing parameters in a model-independent way. Therefore any attempt to fully reconstruct a supersymmetric model from experimental data will rely on assuming some structure for that model. In particular, in the MSSM, the chargino and neutralino masses and couplings depend on five unknown parameters, namely $M_{1}, M_{2},|\mu|, \phi_{\mu}$ and $\tan \beta$. In the MSSM, these parameters can be related to production cross-sections and masses. Thus with a sufficient amount of measurements for chargino and neutralino masses and cross-sections, all the underlying parameters can be extracted.

For the sample scenario BGEN (see appendix), the chargino and neutralino masses at tree-level amount to

$$
\begin{aligned}
& m_{\tilde{\chi}_{1}^{0}}=106.6 \mathrm{GeV}, \quad m_{\tilde{\chi}_{2}^{0}}=170.8 \mathrm{GeV}, \quad m_{\tilde{\chi}_{1}^{ \pm}}=162.7 \mathrm{GeV}, \\
& m_{\tilde{\chi}_{3}^{0}}=231.2 \mathrm{GeV}, \quad m_{\tilde{\chi}_{4}^{0}}=297.7 \mathrm{GeV}, \quad m_{\tilde{\chi}_{2}^{ \pm}}=296.2 \mathrm{GeV} .
\end{aligned}
$$

At the ILC with $\sqrt{s}=500 \mathrm{GeV}$, many neutralino and chargino states are accessible, see Tab. 1. As evident from the table, the most promising processes are $e^{+} e^{-} \rightarrow \tilde{\chi}_{1}^{+} \tilde{\chi}_{1}^{-}$, $e^{+} e^{-} \rightarrow \tilde{\chi}_{1}^{0} \tilde{\chi}_{3}^{0}$ and $e^{+} e^{-} \rightarrow \tilde{\chi}_{2}^{0} \tilde{\chi}_{3}^{0}$. The production of mixed charginos pairs, $e^{+} e^{-} \rightarrow \tilde{\chi}_{1}^{ \pm} \tilde{\chi}_{2}^{\mp}$, also has a sizeable cross-section, but is overwhelmed by background from $e^{+} e^{-} \rightarrow \tilde{\chi}_{1}^{+} \tilde{\chi}_{1}^{-}$.

The relevant decay processes are summarized in Tab. 2. While the two lightest neutralinos dominantly decay into (virtual) $Z$ bosons, the lightest chargino will decay into the light stop and a bottom. For light stops, the by far dominant decay mode is the loop induced process $\tilde{t} \rightarrow c \tilde{\chi}_{1}^{0}[31]$.

\section{Chargino $\tilde{\chi}_{1}^{+}$}

Production of light charginos, $e^{+} e^{-} \rightarrow \tilde{\chi}_{1}^{+} \tilde{\chi}_{1}^{-}$, has a large cross-section of $665 \mathrm{fb}$ at $\sqrt{s}=500$ 


\begin{tabular}{|c|c|c|c|c|}
\hline Sparticle & Mass $m[\mathrm{GeV}]$ & Width $\Gamma[\mathrm{GeV}]$ & \multicolumn{2}{|c|}{ Decay modes } \\
\hline$\tilde{\chi}_{1}^{0}$ & 106.6 & - & \multicolumn{2}{|l|}{ - } \\
\hline$\tilde{\chi}_{2}^{0}$ & 170.8 & 0.00002 & $\tilde{\chi}_{2}^{0} \rightarrow Z^{*} \tilde{\chi}_{1}^{0}$ & $100 \%$ \\
\hline$\tilde{\chi}_{3}^{0}$ & 231.2 & 0.11 & $\tilde{\chi}_{3}^{0} \rightarrow Z \tilde{\chi}_{1}^{0}$ & $98 \%$ \\
\hline \multirow{7}{*}{$\tilde{\chi}_{4}^{0}$} & \multirow{7}{*}{297.7} & & $\rightarrow h^{0} \tilde{\chi}_{1}^{0}$ & $2 \%$ \\
\hline & & \multirow[t]{6}{*}{0.86} & $\tilde{\chi}_{4}^{0} \rightarrow Z \tilde{\chi}_{1}^{0}$ & $1 \%$ \\
\hline & & & $\rightarrow Z \tilde{\chi}_{2}^{0}$ & $\ll 1 \%$ \\
\hline & & & $\rightarrow Z \tilde{\chi}_{3}^{0}$ & $1 \%$ \\
\hline & & & $\rightarrow W^{ \pm} \tilde{\chi}_{1}^{\mp}$ & $94 \%$ \\
\hline & & & $\rightarrow h^{0} \tilde{\chi}_{1}^{0}$ & $4 \%$ \\
\hline & & & $\rightarrow h^{0} \tilde{\chi}_{2}^{0}$ & $\ll 1 \%$ \\
\hline$\tilde{\chi}_{1}^{ \pm}$ & 162.7 & 0.24 & $\tilde{\chi}_{1}^{+} \rightarrow \tilde{t} \bar{b}$ & $100 \%$ \\
\hline \multirow[t]{5}{*}{$\tilde{\chi}_{2}^{ \pm}$} & \multirow[t]{5}{*}{296.2} & \multirow[t]{5}{*}{4.2} & $\tilde{\chi}_{2}^{+} \rightarrow \tilde{t} \bar{b}$ & $84 \%$ \\
\hline & & & $\rightarrow W^{+} \tilde{\chi}_{1}^{0}$ & $\ll 1 \%$ \\
\hline & & & $\rightarrow W^{+} \tilde{\chi}_{2}^{0}$ & $10 \%$ \\
\hline & & & $\rightarrow Z \tilde{\chi}_{1}^{+}$ & \\
\hline & & & $\rightarrow h^{0} \tilde{\chi}_{1}^{+}$ & $\ll 1 \%$ \\
\hline
\end{tabular}

Table 2: Tree-level masses, widths and main branching ratios of the neutralino and chargino states at Born level for the reference point BGEN.

$\mathrm{GeV}$. It can be further increased by using beam polarization, $P\left(e^{+}\right) / P\left(e^{-}\right)=+50 \% /-80 \%$, where + stands for right-handed, - for left-handed polarization, yielding $\sigma\left[e^{+} e^{-} \rightarrow \tilde{\chi}_{1}^{+} \tilde{\chi}_{1}^{-}\right]=$ $1760 \mathrm{fb}$.

The dominant decay chain $e^{+} e^{-} \rightarrow \tilde{\chi}_{1}^{+} \tilde{\chi}_{1}^{-} \rightarrow \tilde{t} \tilde{t}^{*} b \bar{b} \rightarrow c \bar{c} b \bar{b} \tilde{\chi}_{1}^{0} \tilde{\chi}_{1}^{0}$ leads to two charm jets and two bottom jets plus missing energy in the final state. The most important Standard Model backgrounds arise from production of vector bosons in pairs (where the missing energy is generated by mismeasurements) and triples (where neutrino decays can lead to missing energy), as well as $t \bar{t}$ production. In addition, one needs to consider supersymmetric background from neutralino production.

Signal and background events are simulated with the Monte-Carlo methods from Ref. [32], including full tree-level matrix elements and Breit-Wigner propagators for resonant intermediate particles. The processes are generated on the parton level. Jet broadening through parton shower and detector effects are parameterized by smearing functions with lepton and jet energy uncertainty taken from [33]. Jets overlapping within a cone with $\Delta R=\sqrt{\left(\phi_{1}-\phi_{2}\right)^{2}+\left(\eta_{1}-\eta_{2}\right)^{2}}<0.3$ are combined into one jet, where $\phi_{i}$ and $\eta_{i}$ are the azimuthal angle and rapidity of jet $i$. Similarly, a lepton lying within a jet is combined into the jet. Leptons and jets outside the central region of the detector have a higher likelihood of mistag and get inflicted by large two-photon background. Therefore leptons within an 
angle of $|\cos \theta|<0.95$ around the beam line and jets with $|\cos \theta|<0.90$ are discarded. After these numerical adjustments the remaining isolated jets and leptons define the signature of the simulated event.

Background from two-photon interactions was not simulated for this study, since they typically lead to very soft hadronic events, and can be removed by a cut on the total transverse momentum, $p_{\mathrm{t}}^{\text {tot }}>12 \mathrm{GeV}[9]$.

The other backgrounds are reduced by the following simple kinematic cuts:

- Each event must contain four hadronic jets and no isolated lepton and $p_{\mathrm{t}}^{\text {tot }}>12 \mathrm{GeV}$ (see above).

- For each combination of two jets, the invariant mass is required to be different from the $Z$ mass, $\left|m_{j j}-m_{\mathrm{Z}}\right|>10 \mathrm{GeV}$. This strongly reduces background from gauge bosons and neutralinos.

- Since backgrounds from gauge bosons tend to increase in the forward and backward detector regions, they can be further reduced by a cut on total momentum angle, $\left|\cos \theta_{p_{\text {tot }}}\right|=\left|p_{\text {long,tot }} / p_{\text {tot }}\right|<0.9$.

- All Standard Model backgrounds are cut down by requiring large missing energy, मॄ > $100 \mathrm{GeV}$.

- Since two bottom jets are expected in the signal, the signal-to-background ratio is improved through b-tagging. Following [34], it is assumed that the b tagging efficiency is $90 \%$, with a mistag probability of light flavors of $10 \%$. B-tagging works on all backgrounds except $t \bar{t}$. For the other backgrounds the four jets mainly originate from $W$ decays, so that two of the jets could be charmed. Charm jets have a higher bmistagging probability than light flavors, but since the signal also always contains two charm jets, the resulting tagging power between signal and gauge boson backgrounds is governed by the discrimination between bottom and light flavors.

With this selection procedure, the remaining background is very small, about $3 \mathrm{fb}$, whereas the total signal efficiency is $18 \%$. Including an overall systematic acceptance of $90 \%$, the resulting signal efficiency is $16 \%$. With a total luminosity of $250 \mathrm{fb}^{-1}$ the statistical error for the cross-section measurements is

$$
\delta \sigma_{\mathrm{RL}}\left[\chi_{1}^{+} \tilde{\chi}_{1}^{-}\right]=0.37 \%, \quad \delta \sigma_{\mathrm{LR}}\left[\chi_{1}^{+} \tilde{\chi}_{1}^{-}\right]=1.6 \%
$$

where RL and LR stand for the polarization combinations $P\left(e^{+}\right) / P\left(e^{-}\right)=+50 \% /-80 \%$ and $-50 \% /+80 \%$, respectively.

The spectrum of the decay products can be used for a determination of the chargino and neutralino mass. The spectrum of the invariant mass of the $\mathrm{b}$ and $\mathrm{c}$ jet from the decay $\tilde{\chi}_{1}^{+} \rightarrow \tilde{t} \bar{b} \rightarrow c \bar{b} \tilde{\chi}_{1}^{0}$ has a characteristic upper edge at

$$
m_{\overline{\mathrm{bc}}, \max }=m_{\mathrm{b} \bar{c}, \max }=m_{\tilde{\chi}_{1}^{ \pm}}-m_{\tilde{\chi}_{1}^{0}} .
$$




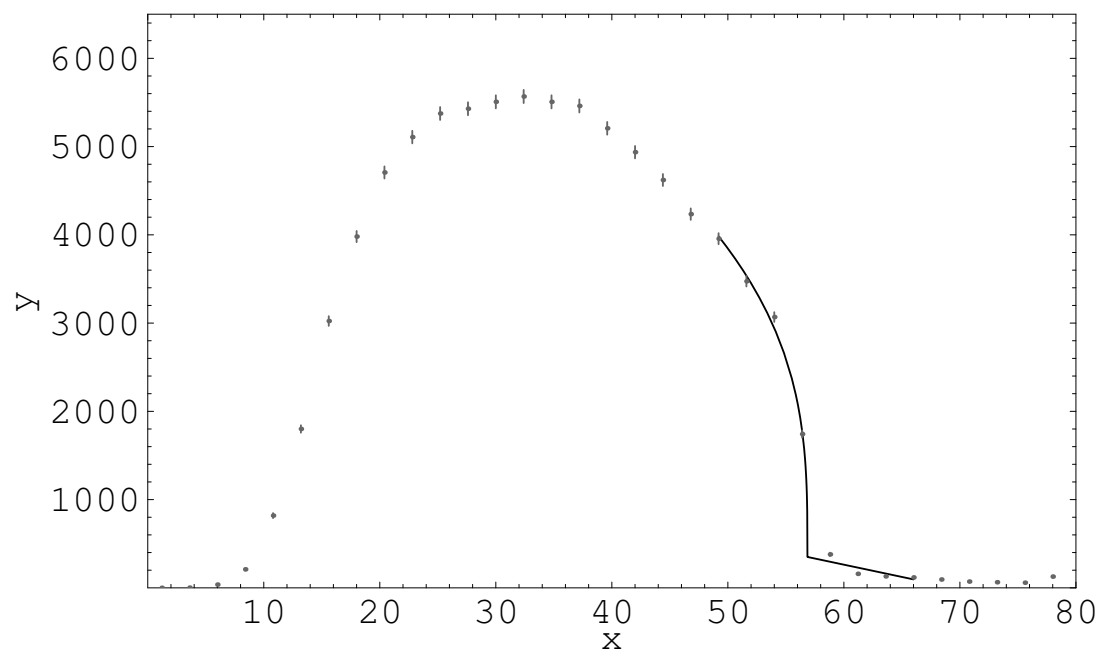

Figure 1: Distribution of the bottom-charm invariant mass $m_{\mathrm{bc}}$ from chargino decay and a simple fit to the upper edge.

However, with a four jet final state the problem remains to identify the pairs of two jets each that belong to the decay of one chargino. The bottom jets can be identified as the jets with the highest $\mathrm{b}$ tagging likelihood. However this still leaves a twofold ambiguity to combine $\mathrm{a} b$ jet with a $\mathrm{c}$ jet. Here the following strategy is adopted: since a pair of jets originating from different charginos tend to have a larger invariant mass than a pair originating from the same chargino, always the pair with the lower invariant mass is selected for the mass measurement.

The resulting invariant mass distribution in shown in Fig. 1. A very crude fit of the upper threshold gives

$$
m_{\mathrm{bc}, \max }=\left(56.6_{-0.06}^{+0.22} \pm 0.24\right) \mathrm{GeV},
$$

compared to the model input value $m_{\tilde{\chi}_{1}^{ \pm}}-m_{\tilde{\chi}_{1}^{0}}=56.8 \mathrm{GeV}$. Here the first error is the statistical uncertainty of the fit, whereas the second error indicates the systematic uncertainty stemming from the jet energy scale. Based on studies at LEP for $W$ boson pair production [35], the jet energy scale error is assumed to be $0.4 \%$.

Since this measurement would only yield the difference between chargino and neutralino mass, an independent measurement is necessary for the determination of absolute values of both masses. A very precise direct determination of the chargino mass can be obtained from a threshold scan. By measuring the chargino cross-section at a few center-of-mass energies near the pair production threshold, the onset of the pair production excitation curve can be reconstructed and used for a mass determination. By including two points below the threshold, the background can be analyzed and extrapolated in a model-independent way. The result of the analysis for five scan points with $10 \mathrm{fb}^{-1}$ luminosity each, using the same methods and selection cuts as above, is depicted in Fig. 2. The result of a fit to the threshold 


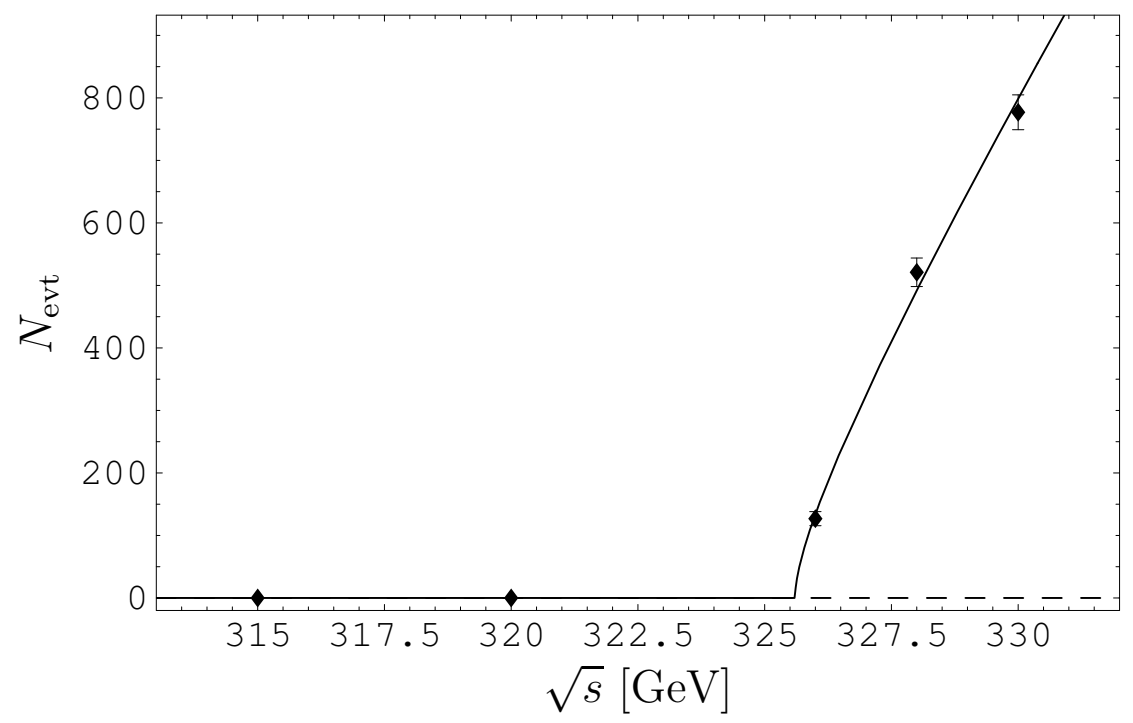

Figure 2: Threshold scan for chargino pair production using five scan points with $10 \mathrm{fb}^{-1}$ each.

excitation curve is

$$
m_{\tilde{\chi}_{1}^{ \pm}}=\frac{1}{2}[\sqrt{s}]_{\mathrm{thr}}=(163.02 \pm 0.04 \pm 0.04) \mathrm{GeV},
$$

where the first error is statistical and the second is the systematic error from the beam energy calibration. Combining eqs. (44) and (5), the masses are obtained as follows,

$$
m_{\tilde{\chi}_{1}^{ \pm}}=(163.02 \pm 0.06) \mathrm{GeV}, \quad m_{\tilde{\chi}_{1}^{0}}=(106.1 \pm 0.3) .
$$

\section{Neutralinos $\tilde{\chi}_{2}^{0}$ and $\tilde{\chi}_{3}^{0}$}

Neutralino production can have sizeable cross-section for mixed pair production of $\tilde{\chi}_{1}^{0} \tilde{\chi}_{3}^{0}$ and $\tilde{\chi}_{2}^{0} \tilde{\chi}_{3}^{0}$. The signal for $\tilde{\chi}_{1}^{0} \tilde{\chi}_{3}^{0}$ is characterized by two leptons or two jets plus missing energy, which receives very large backgrounds from $W^{+} W^{-}$and $Z Z$ production. Even after reducing the backgrounds with appropriate cuts, the remaining level is still problematic for any precision measurement. Therefore, in the following only $\tilde{\chi}_{2}^{0} \tilde{\chi}_{3}^{0}$ is considered.

This process has a sizeable cross-section, $\sigma\left[e^{+} e^{-} \rightarrow \tilde{\chi}_{2}^{0} \tilde{\chi}_{3}^{0}\right]=62 \mathrm{fb}$ for unpolarized beams. Using right-handed polarization for the $e^{-}$and left-handed polarization for the $e^{+}$beam, $P\left(e^{+}\right) / P\left(e^{-}\right)=-50 \% /+80 \%$, the signal is enhanced to $68.9 \mathrm{fb}$, while all Standard Model backgrounds, which are dominated by left-chiral $\mathrm{SU}(2)$ interactions, are reduced for this beam polarization combination.

The $\tilde{\chi}_{3}^{0}$ almost always decays into a $Z$ boson and lightest neutralino $\tilde{\chi}_{1}^{0}$, while the $\tilde{\chi}_{2}^{0}$, due to the small mass difference $m_{\tilde{\chi}_{2}^{0}}-m_{\tilde{\chi}_{1}^{0}}$ decays through a virtual $Z$ into two quarks or two leptons. In order to improve the statistical significance of the signal, the hadronic decay modes of the $Z$ are considered, since the branching ratio of $Z$ to leptons is very small. Then the final state is characterized by four (light quark) jets and missing energy. Standard Model backgrounds arise from processes with two and three vector bosons and from $t \bar{t}$ production. In addition, chargino production as discussed above, constitutes another difficult background. 
Signal and background are simulated and analyzed as detailed above. The following cuts have been applied to select the signal out of the background:

- Similar to the chargino analysis, a four jets signature and the same cuts on $\cos \theta_{p_{\text {tot }}}$ and $E$ ' are used.

- The invariant mass of two jets has to be equal to the $Z$ boson mass, $\left|m_{j_{a} j_{b}}-m_{\mathrm{Z}}\right|<10$ $\mathrm{GeV}$, whereas the invariant mass of the other two jets has to be smaller than $m_{\mathrm{Z}}$, $m_{\mathrm{Z}}-m_{j_{c} j_{d}}>10 \mathrm{GeV}$. All combinatoric pairings of the four jets are tried for this purpose, and if one combination meets these requirement, the event is kept.

- The Background from charginos generates rather soft jets due to the small stop mass. Thus a cut on the transverse momentum, $p_{\mathrm{t}}^{\text {tot }}>50 \mathrm{GeV}$ is effective against this background.

- Finally the chargino background is further reduced by a b-quark veto. As a consequence, here the signal is restricted to light quark decay channels of the $Z$ bosons only.

After this selection procedure, the remaining background is around $0.2 \mathrm{fb}$, but a good signal efficiency of $26 \%$ is achieved. Including a general systematic acceptance of $90 \%$, the resulting signal efficiency is $24 \%$. With a total luminosity of $250 \mathrm{fb}^{-1}$ for the polarization combination $P\left(e^{+}\right) / P\left(e^{-}\right)=-50 \% /+80 \%$, the statistical error for the cross-section measurement is

$$
\delta \sigma_{\mathrm{RL}}\left[\tilde{\chi}_{2}^{0} \tilde{\chi}_{3}^{0}\right]=1.6 \%
$$

For the opposite polarization combination $P\left(e^{+}\right) / P\left(e^{-}\right)=-50 \% /+80 \%$, the precision of the neutralino cross-section measurement is much worse due to larger backgrounds, and does not improve the global MSSM analysis.

Information about the neutralino masses can be extracted from the decay product distributions. In the $\tilde{\chi}_{3}^{0} \rightarrow Z \tilde{\chi}_{1}^{0}$ decay, the energy spectrum of the $Z$ is rather flat, with distinct lower and upper endpoints at

$$
\begin{aligned}
E_{\min , \max , 3}=\frac{1}{4 m_{\tilde{\chi}_{3}^{0}}^{2} \sqrt{s}}( & m_{\tilde{\chi}_{3}^{0}}^{4}-m_{\tilde{\chi}_{3}^{0}}^{2} m_{\tilde{\chi}_{2}^{0}}^{2}+m_{\tilde{\chi}_{3}^{0}}^{2} m_{\mathrm{Z}}^{2}-m_{\tilde{\chi}_{2}^{0}}^{2} m_{\mathrm{Z}}^{2}+m_{\tilde{\chi}_{3}^{0}}^{2} s+m_{\mathrm{Z}^{2}}^{2} s \\
& \left.\quad m_{\tilde{\chi}_{1}^{0}}^{2}\left(m_{\tilde{\chi}_{3}^{0}}^{2}-m_{\tilde{\chi}_{2}^{0}}^{2}+s\right) \mp \sqrt{\lambda\left(m_{\tilde{\chi}_{3}^{0}}^{2}, m_{\tilde{\chi}_{1}^{0}}^{2}, m_{\mathrm{Z}}^{2}\right) \lambda\left(m_{\tilde{\chi}_{3}^{0}}^{2}, m_{\tilde{\chi}_{2}^{0}}^{2}, s\right)}\right),
\end{aligned}
$$

with $\lambda(a, b, c)=a^{2}+b^{2}+c^{2}-2 a b-2 a c-2 b c$. The energy of the $Z$ boson can be deduced from the energy of the jet pair that combines to the $Z$ invariant mass.

For the $\tilde{\chi}_{2}^{0}$ decay, the kinematics are different since only a virtual $Z$ is involved in the process. Still the energy spectrum of the two final state jets has a distinct upper endpoint given by

$$
E_{\mathrm{jj}, \max , 2}=\frac{m_{\tilde{\chi}_{2}^{0}}^{2}-m_{\tilde{\chi}_{3}^{0}}^{2}-2 m_{\tilde{\chi}_{1}^{0}}^{2} \sqrt{s}+s}{2 \sqrt{s}} .
$$


(a)

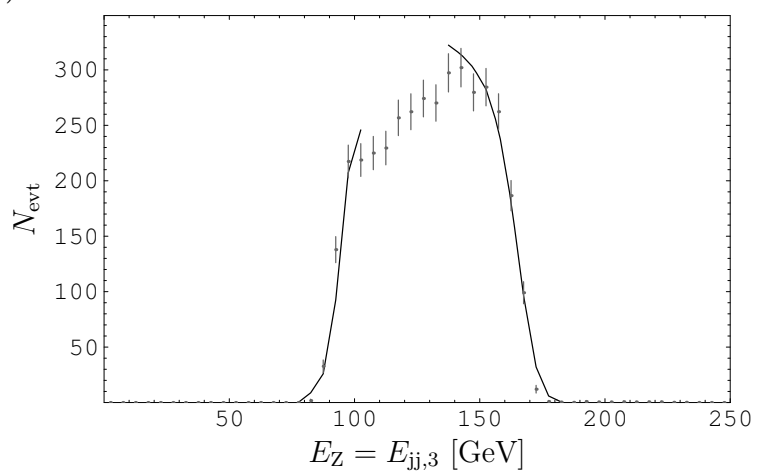

(c)

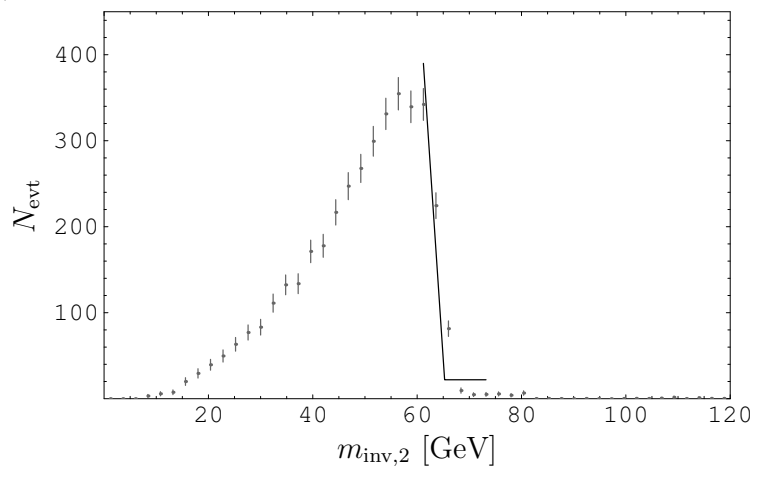

(b)

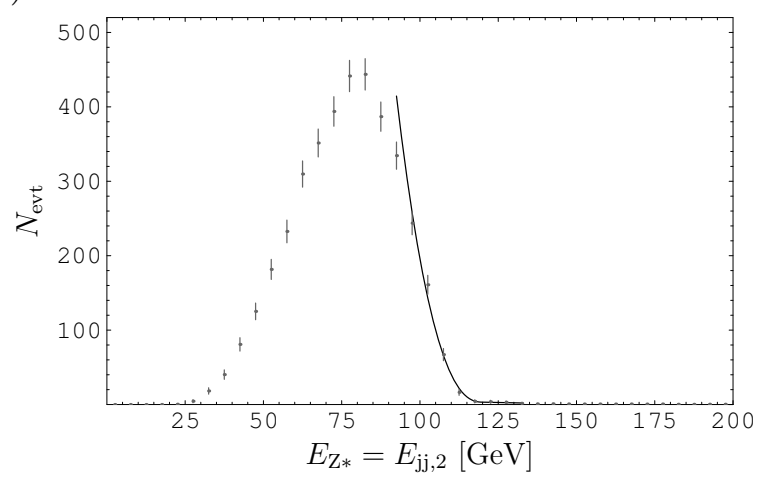

Figure 3: (a) Energy distribution for jet pair in $\tilde{\chi}_{3}^{0} \rightarrow Z \tilde{\chi}_{1}^{0}$ decay, (b) energy distribution for jet pair in $\tilde{\chi}_{2}^{0} \rightarrow Z^{*} \tilde{\chi}_{1}^{0}$ decay, and (c) invariant mass of the latter, including simple fitting curves.

In addition, the invariant mass of the same two jets is bounded from above by the mass difference of the $\tilde{\chi}_{2}^{0}$ and $\tilde{\chi}_{1}^{0}$,

$$
m_{\mathrm{jj}, \max , 2}=m_{\tilde{\chi}_{2}^{0}}-m_{\tilde{\chi}_{1}^{0}} .
$$

The simulated distributions after cuts and fits to the endpoints are summarized in Fig. [3. From a combination of the different fits, and including the jet energy scale error as for the charginos, one obtains

$$
\delta m_{\tilde{\chi}_{1}^{0}}=1.3 \mathrm{GeV}, \quad \delta m_{\tilde{\chi}_{2}^{0}}=1.5 \mathrm{GeV}, \quad \delta m_{\tilde{\chi}_{3}^{0}} \approx 3.3 \mathrm{GeV} .
$$

By feeding in the more precise determination of the lightest neutralino mass in eq. (6), these numbers are improved to

$$
\delta m_{\tilde{\chi}_{2}^{0}}=0.6 \mathrm{GeV}, \quad \delta m_{\tilde{\chi}_{3}^{0}} \approx 2.0 \mathrm{GeV} .
$$

The mass measurements are not sufficient to extract the fundamental supersymmetry parameters without ambiguity. Therefore the additional information from measurements of the chargino and neutralino cross-sections are important. The MSSM model parameters can be extracted from a combined fit to all observables.

As a result of very heavy selectrons and sneutrinos, the t-channel contribution to the production processes are essentially switched off. However, the non-observation of these particles at colliders puts only a relatively mild lower bound on their masses of about 500 
$\mathrm{GeV}$. Here the values of the sleptons masses are kept arbritrary, and instead indirect bounds on the masses are derived from the fit (see also Ref. [30]).

The chargino and neutralino cross-section and mass measurements are combined in a $\chi^{2}$ fit, thus taking into account all correlations in the underlying parameters that enter in the observables. The fit results with one standard deviation errors are

$$
\begin{aligned}
M_{1} & =(118.8 \pm 0.4) \mathrm{GeV}, & & \tan \beta=5_{-0.7}^{+0.5} \\
M_{2} & =(225.0 \pm 0.9) \mathrm{GeV}, & & \\
|\mu| & =(225.0 \pm 1.2) \mathrm{GeV}, & & m_{\tilde{\nu}_{\mathrm{e}}}>5 \mathrm{TeV} \\
\left|\phi_{\mu}\right| & <0.6, & & m_{\tilde{e}_{\mathrm{R}}}>1.5 \mathrm{TeV} .
\end{aligned}
$$

The resulting constraint on the phase $\phi_{\mu}$ is rather poor, since none of the included observables is directly CP-sensitive, and $\phi_{\mu}$ is strongly correlated with other parameters, in particular $\tan \beta$.

\section{CP violation}

The effect of CP violation can be uniquely studied in observables that are directly CPsensitive. In the chargino and neutralino sector, triple products of kinematic momenta have been identified as useful for that purpose [36-38].

In this work, hadronic decays of neutralinos and charginos are investigated,

$$
\begin{aligned}
& e^{+} e^{-} \rightarrow \tilde{\chi}_{i}^{s} \tilde{\chi}_{j}^{-s} \quad s=0, \pm . \\
& \longrightarrow j_{\mathrm{a}} j_{\mathrm{b}} \tilde{\chi}_{1}^{0}
\end{aligned}
$$

From the momenta $p_{\mathrm{a}, \mathrm{b}}$ of the two jets one can construct the T-odd triple product

$$
\mathcal{T}=\vec{p}_{\mathrm{e}^{-}}\left(\vec{p}_{\mathrm{a}} \times \vec{p}_{\mathrm{b}}\right)
$$

and the T-odd asymmetry

$$
\mathcal{A}=\frac{\sigma[\mathcal{T}>0]-\sigma[\mathcal{T}<0]}{\sigma[\mathcal{T}>0]+\sigma[\mathcal{T}<0]}
$$

The experimental investigation of this triple product requires the identification of the charge of the jets. While this is not possible for light-quark jets on a event-by-event basis, it can be achieved for larger samples of jet events on a statistical basis, see e.g. Ref. [39].

Using the same Monte-Carlo techniques as explained above, the asymmetry is calculated both for chargino and neutralino production. In the given scenario, it is found that $\mathcal{A}\left[\tilde{\chi}_{1}^{+} \tilde{\chi}_{1}^{-}\right]=0.3 \%$ and $\mathcal{A}\left[\tilde{\chi}_{2}^{0} \tilde{\chi}_{3}^{0}\right]=0.9 \%$. Thus in both cases the expected magnitude is of the same order as the statistical error of the cross-section measurements, see eqs. (2), (77).

For larger values of $\phi_{\mu}$, larger triple product asymmetries can be obtained, however such models are already highly constrained by electric dipole moment limits. Therefore a measurement of the $\mathrm{CP}$-violating phase in the $\mu$ parameter appears to be hopeless at the ILC. These findings are in agreement with previous theoretical studies [37]. 


\subsection{Cosmological implications}

The discovery of light scalar top quarks, in conjunction with a Standard-Model-like Higgs boson with a mass near $120 \mathrm{GeV}$, would be a strong indication that electroweak baryogenesis is the mechanism for the generation of the baryon asymmetry. At the same time, supersymmetry could also explain the existence of dark matter in the universe, based on the co-annihilation mechanism. In order to confirm this exciting picture, the relevant supersymmetry parameters have to be measured accurately.

One needs to (i) determine that the light stop is mainly right-chiral to contribute appropriately to the electroweak phase transition while being in agreement with electroweak precision measurements, (ii) check that the masses and compositions of the gauge/Higgs superfield sector are compatible with the values required for the generation of the baryon asymmetry, and (iii) compute the dark matter annihilation cross-sections and the relic abundance so as to compare with cosmological observations. If stop-neutralino co-annihilation is relevant it is important to determine the stop-neutralino mass difference very precisely.

\section{Baryogenesis}

In the given scenario, the first information about the necessary constituents for baryogenesis in the MSSM can be obtained at the LHC, namely the discovery of a light Higgs boson and possibly evidence for a light stop. At the ILC, the picture can be rendered much more precisely by determining the mass and mixing angle of the light stop accurately.

Using the computations of Refs. [5,20] for the electroweak phase transition, it is found that the experimental uncertainty of the stop parameters allows to determine the strength of the phase transition to better than $10 \%$,

$$
\delta_{\exp }\left[\frac{v\left(T_{\mathrm{c}}\right)}{T_{\mathrm{c}}}\right] \lesssim 10 \% .
$$

Note however that this is only a parametric error, and there are also theoretical uncertainties involved in the computation.

Besides the strength of the electroweak phase transition, the second crucial ingredient for electroweak baryogenesis is the generation of the baryon asymmetry through CP-violating processes. As explained in section 2, in the MSSM these CP-violating contributions can be described by a complex phase of the parameter $\mu$. However, the findings of the previous sections show that for typical values of $\phi_{\mu}$, the CP-violating effects in neutralino and chargino observables at the ILC are too small to be observed. It would only be possible to derive an upper bound of $\left|\phi_{\mu}\right| \lesssim 0.7$ at the $90 \%$ confidence level, which would leave the question of the baryon asymmetry still undecided.

\section{Dark matter}

As discussed widely in the literature (see e.g. $[9,28,40]$ ), collider experiment data can be used to compute the expected cosmological dark matter relic density. In the scenario studied here, the relic density is governed by the neutralino-stop co-annihilation mechanism, and thus

crucially depends on the stop-neutralino mass difference. It was found [41] that this mass 


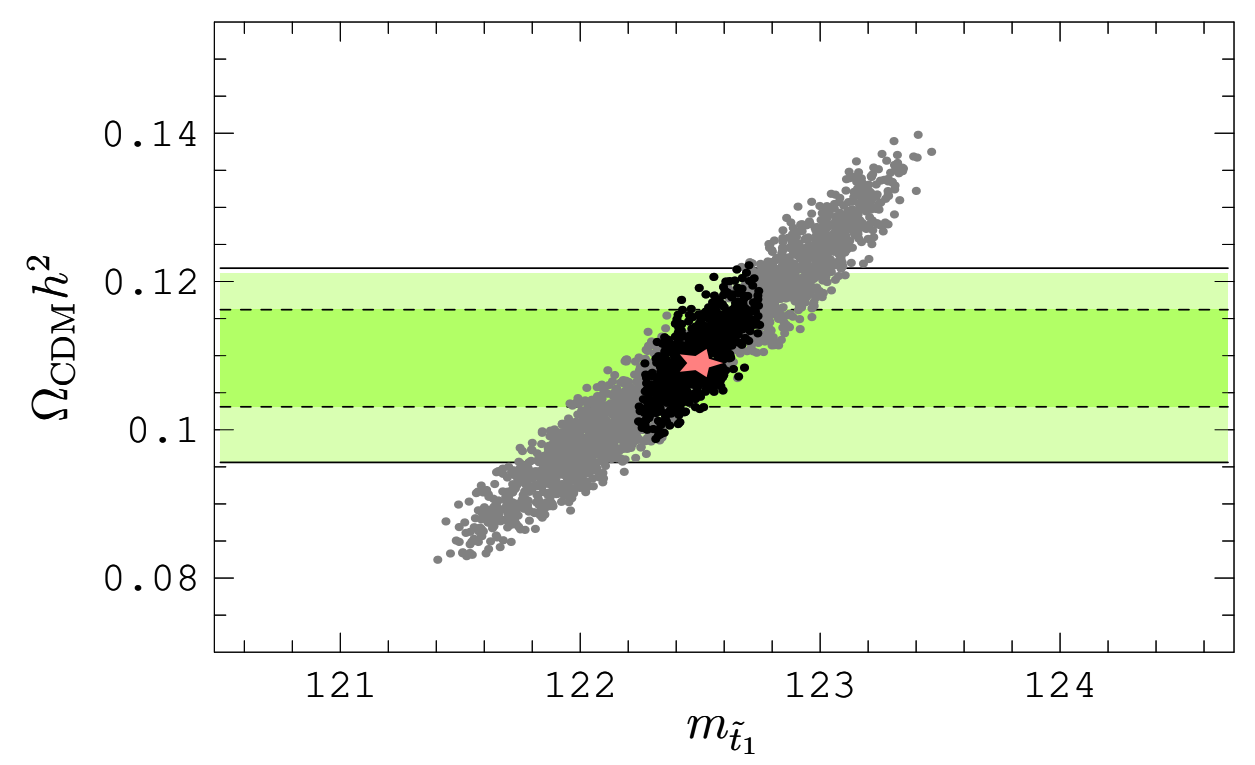

Figure 4: Computation of dark matter relic abundance $\Omega_{\mathrm{CDM}} h^{2}$ for the scenario BGEN, taking into account estimated experimental errors for stop, chargino, neutralino and Higgs sector measurements at future colliders. The dots correspond to a scan over the $1 \sigma\left(\Delta \chi^{2} \leq 1\right)$ region allowed by the experimental errors, as a function of the measured stop mass, for a mass measurement error of $1.2 \mathrm{GeV}$ (gray dots) and $0.3 \mathrm{GeV}$ (black dots). The original scenario used as input is indicated by the red (light gray) star. The horizontal shaded bands show the $1 \sigma$ and $2 \sigma$ constraints on the relic density measured by WMAP and SDSS.

difference cannot be extracted with good precision directly from stop decay distributions, because of radiation effects and limited statistics, but the best determination is derived from independent mass measurements of the stop and neutralino. While the stop mass and mixings can be extracted from precision measurements of the stop cross-section [9], the neutralino properties have to be determined from independent observables as discussed in this report.

The relic dark matter density is computed with the codes described in Ref. [12, 22]. The analysis is based on observables in the stop and neutralino/chargino sectors. For the neutralinos and charginos, the estimated errors taken from the previous chapter, see eqs. (22), (66), (7), (12). The stop measurement errors are derived from the study of Ref. [9] with the result: $\delta m_{\tilde{t}_{1}}=1.2 \mathrm{GeV}$ and $\left|\cos \theta_{\tilde{t}}\right|<0.077$.

In total, the relevant parameters used as input are $m_{\tilde{t}_{1}}, \cos \theta_{\tilde{t}}, M_{1}, M_{2},|\mu|, \phi_{\mu}, \tan \beta$. It has been checked that the dependence on Higgs and slepton parameters is negligible for this scenario. The mass of the heavier stop $\tilde{t}_{2}$ is too large to be measured directly, but it is supposed that a limit of $m_{\tilde{t}_{2}}>1000 \mathrm{GeV}$ can be set from LHC searches.

The expected experimental errors are propagated and parametric correlations are taken into account by means of a $\chi^{2}$ scan. However, theoretical errors due to missing radiative corrections are not taken into account in the calculation of the dark matter density. Fig. 目 shows the result of a scan over the MSSM parameter space for the scenario BGEN. The 
scattered gray dots indicate the region allowed by the collider experimental uncertainty, as a function of the measured stop mass. The range of the horizontal axis is constrained by the error in the stop mass measurement, $m_{\tilde{t}_{1}}=(122.5 \pm 1.2) \mathrm{GeV}$. The horizontal bands depict the relic density as measured by WMAP and SDSS $[1,2]$ with one and two standard deviation errors. At $1 \sigma$ level, the astrophysical observations lead to $0.103<\Omega_{\mathrm{CDM}} h^{2}<0.116$.

In total, using the collider measurement simulations, the relic density can be predicted to $0.082<\Omega_{\mathrm{CDM}} h^{2}<0.139$ at the $1 \sigma$ level. Thus the overall precision is of the same magnitude as, but worse by roughly a factor 4 than the direct WMAP/SDSS determination. The uncertainty in the theoretical determination is dominated by the measurement of the $\tilde{t}_{1}$ mass with an error of $1.2 \mathrm{GeV}$. This shows that even in a scenario with heavy sfermions, which is unfavorable for future collider experiments, the achievable precision in the analysis of the chargino and neutralino sector is very high and not a limiting factor for cosmological interpretations.

First results of an optimized threshold scan method indicate that the precision for $m_{\tilde{t}_{1}}$ can be improved to about $0.3 \mathrm{GeV}$ [42]. The advantage of the threshold scan method is the small influence of systematic errors, since it makes use of the shape of the cross-section as a function of the center-of-mass energy, instead of absolute cross-section measurements. However it is limited by small statistics near the threshold. The best accuracy can be achieved by combining a measurement near the threshold, where the cross-section is most sensitive to the stop mass, with a measurement at higher energies, where the cross-section is larger [42]. With a stop mass error of $\delta m_{\tilde{t}_{1}}=0.3 \mathrm{GeV}$, the relic density could be computed much more precisely, yielding the result $0.099<\Omega_{\mathrm{CDM}} h^{2}<0.122$ in the scenario BGEN. This precision is very comparable to the direct WMAP/SDSS determination, as indicated by the black dots in Fig. 4 .

\section{Focus-point scenario}

\subsection{Chargino and neutralino studies at the LHC}

In a focus-point scenario, all sfermions are very heavy, but gluinos $\tilde{g}$ can be sufficiently light to be generated with large cross-sections. The gluino decays can proceed via some neutralinos and charginos, giving a clear and distinct leptonic discovery signature [29]. For example, a signature with same-sign leptons and missing transverse energy can be interpreted as a decay cascade of the gluinos via charginos [43]. Besides, the decay $\tilde{\chi}_{2}^{0} \rightarrow Z^{*} \tilde{\chi}_{1}^{0} \rightarrow l^{+} l^{-} \tilde{\chi}_{1}^{0}$ allows to determine the mass difference $m_{21}=m_{\tilde{\chi}_{2}^{0}}-m_{\tilde{\chi}_{1}^{0}}$ from the $l^{+} l^{-}$invariant mass distribution with a precision of about $\delta m_{21} \sim(0.5 \mathrm{GeV}) \times \exp \left(-m_{\tilde{g}} /(200 \mathrm{GeV})+3.5\right)$, where the exponential factor with $m_{\tilde{g}}$ approximately accounts for the decrease of the gluino cross-section with larger gluino masses.

\subsection{Chargino and neutralino studies at the ILC}

In the scenario LCC2, among the superpartners, only charginos and neutralinos can be studied at a future linear collider, whereas all sfermions are too massive to be accessible 


\begin{tabular}{|r||rrr|}
\hline$e^{+} e^{-} \rightarrow \tilde{\chi}_{i}^{0} \tilde{\chi}_{j}^{0}$ & $\tilde{\chi}_{i}^{0}=\tilde{\chi}_{2}^{0}$ & $\tilde{\chi}_{3}^{0}$ & $\tilde{\chi}_{4}^{0}$ \\
\hline \hline$\tilde{\chi}_{j}^{0}=\tilde{\chi}_{1}^{0}$ & 8.0 & 46.3 & 0.01 \\
$\tilde{\chi}_{2}^{0}$ & 8.7 & 107.2 & 0.07 \\
$\tilde{\chi}_{3}^{0}$ & & 6.2 & 22.7 \\
$\tilde{\chi}_{4}^{0}$ & & & - \\
\hline \hline$e^{+} e^{-} \rightarrow \tilde{\chi}_{i}^{ \pm} \tilde{\chi}_{j}^{\mp}$ & $\tilde{\chi}_{i}^{ \pm}=\tilde{\chi}_{1}^{ \pm}$ & $\tilde{\chi}_{2}^{ \pm}$ & \\
\hline \hline$\tilde{\chi}_{j}^{\mp}=\tilde{\chi}_{1}^{\mp}$ & 534 & 23 & \\
$\tilde{\chi}_{2}^{\mp}$ & & - & \\
\hline
\end{tabular}

Table 3: Tree-level production cross-sections in fb at $\sqrt{s}=500 \mathrm{GeV}$ with unpolarized beams for the reference point LCC2.

either at the LHC or ILC. This situation arises always in focus point supersymmetry and has been studied in previous works [27,30]. Here the study of Ref. [30] is extended by including different observables and simulations for signal and background processes.

As in the previous section, the general procedure for reconstructing the underlying MSSM parameters relies on the assumption of the MSSM structure for the chargino and neutralino mass matrices. The parameters $M_{1}, M_{2},|\mu|, \phi_{\mu}$ and $\tan \beta$ are extracted from a fit to mass and cross-section measurements.

For the scenario LCC2 (see appendix), the chargino and neutralino masses at tree-level amount to

$$
\begin{aligned}
& m_{\tilde{\chi}_{1}^{0}}=107.7 \mathrm{GeV}, \quad m_{\tilde{\chi}_{2}^{0}}=166.3 \mathrm{GeV}, \quad m_{\tilde{\chi}_{1}^{ \pm}}=159.4 \mathrm{GeV}, \\
& m_{\tilde{\chi}_{3}^{0}}=190.0 \mathrm{GeV}, \quad m_{\tilde{\chi}_{4}^{0}}=294.4 \mathrm{GeV}, \quad m_{\tilde{\chi}_{2}^{ \pm}}=286.8 \mathrm{GeV} .
\end{aligned}
$$

As pointed out above, the LHC has a good possibility to find evidence for and measure some properties of neutralinos and charginos. At the ILC with $\sqrt{s}=500 \mathrm{GeV}$, many neutralino and chargino states are accessible, see Tab. 3. The most promising processes are $e^{+} e^{-} \rightarrow \tilde{\chi}_{1}^{+} \tilde{\chi}_{1}^{-}, e^{+} e^{-} \rightarrow \tilde{\chi}_{1}^{0} \tilde{\chi}_{3}^{0}$ and $e^{+} e^{-} \rightarrow \tilde{\chi}_{2}^{0} \tilde{\chi}_{3}^{0}$, similar to the baryogenesis scenario.

Tab. 4 gives the relevant decay modes and branching fractions. In this scenario, the mass differences between the lighter neutralino and chargino states are rather small, thus allowing only decays of the light states through virtual gauge bosons.

\section{Chargino $\tilde{\chi}_{1}^{+}$}

Production of light charginos, $e^{+} e^{-} \rightarrow \tilde{\chi}_{1}^{+} \tilde{\chi}_{1}^{-}$, has a large cross-section of $534 \mathrm{fb}$ at $\sqrt{s}=500$ $\mathrm{GeV}$, which can be further increased to $1345 \mathrm{fb}$ by using beam polarization, $P\left(e^{+}\right) / P\left(e^{-}\right)$ $=+50 \% /-80 \%$.

In the focus-point scenario, charginos almost always decay through virtual $W$ bosons, $e^{+} e^{-} \rightarrow \tilde{\chi}_{1}^{+} \tilde{\chi}_{1}^{-} \rightarrow W^{+*} W^{-*} \tilde{\chi}_{1}^{0} \tilde{\chi}_{1}^{0} \rightarrow l^{ \pm} \nu_{l} q \bar{q}^{\prime} \tilde{\chi}_{1}^{0} \tilde{\chi}_{1}^{0}$ where it is useful to consider a final state where one $W^{*}$ decays hadronically into quarks, while the other decays into a lepton $l=e, \mu$ and a neutrino. This final state allows a precise kinematical measurement, since the two intermediate virtual $W$ bosons can be disentangled, as opposed to the purely hadronic 


\begin{tabular}{|c||c|c|cr|}
\hline Sparticle & Mass $m[\mathrm{GeV}]$ & Width $\Gamma[\mathrm{GeV}]$ & \multicolumn{3}{|c|}{ Decay modes } \\
\hline \hline$\tilde{\chi}_{1}^{0}$ & 107.7 & - & - & \\
$\tilde{\chi}_{2}^{0}$ & 166.3 & 0.000016 & $\tilde{\chi}_{2}^{0} \rightarrow Z^{*} \tilde{\chi}_{1}^{0}$ & $100 \%$ \\
$\tilde{\chi}_{3}^{0}$ & 190.0 & 0.0013 & $\tilde{\chi}_{3}^{0} \rightarrow Z^{*} \tilde{\chi}_{1}^{0}$ & $100 \%$ \\
& & & $\rightarrow h^{0 *} \tilde{\chi}_{1}^{0}$ & $<1 \%$ \\
$\tilde{\chi}_{4}^{0}$ & 294.4 & 0.81 & $\tilde{\chi}_{4}^{0} \rightarrow Z \tilde{\chi}_{1}^{0}$ & $\ll 1 \%$ \\
& & & $\rightarrow Z \tilde{\chi}_{2}^{0}$ & $1 \%$ \\
& & & $\rightarrow Z \tilde{\chi}_{3}^{0}$ & $1 \%$ \\
& & & $\rightarrow W^{ \pm} \tilde{\chi}_{1}^{\mp}$ & $94 \%$ \\
& & & $\rightarrow h^{0} \tilde{\chi}_{1}^{0}$ & $\ll 1 \%$ \\
& & & $\rightarrow h^{0} \tilde{\chi}_{2}^{0}$ & $3 \%$ \\
\hline$\tilde{\chi}_{1}^{ \pm}$ & 159.4 & 0.0002 & $\tilde{\chi}_{1}^{+} \rightarrow W^{+*} \tilde{\chi}_{1}^{0}$ & $100 \%$ \\
$\tilde{\chi}_{2}^{ \pm}$ & 286.8 & 0.73 & $\tilde{\chi}_{2}^{+} \rightarrow W^{+} \tilde{\chi}_{1}^{0}$ & $3 \%$ \\
& & & $\rightarrow W^{+} \tilde{\chi}_{2}^{0}$ & $42 \%$ \\
& & & $\rightarrow W^{+} \tilde{\chi}_{3}^{0}$ & $11 \%$ \\
& & & $\rightarrow Z \tilde{\chi}_{1}^{+}$ & $36 \%$ \\
& & & $\rightarrow h^{0} \tilde{\chi}_{1}^{+}$ & $8 \%$ \\
\hline
\end{tabular}

Table 4: Tree-level masses, widths and main branching ratios of the neutralino and chargino states at Born level for the reference point LCC2.

final state. As before, Standard Model background from vector bosons and $t \bar{t}$, as well as supersymmetric background from neutralino production is taken into account.

Signal and background events are simulated with the same Monte-Carlo methods as elaborated above. The following selection cuts are applied to reduce the backgrounds:

- Each event must contain two hadronic jets, one isolated lepton and $p_{\mathrm{t}}^{\text {tot }}>12 \mathrm{GeV}$.

- The invariant mass of the two jets is required to be smaller than the $W$ mass, $m_{\mathrm{W}}-$ $m_{j j}<10 \mathrm{GeV}$, to reduce Standard Model background.

- All Standard Model backgrounds are cut down by requiring large missing energy, \#゙ > $100 \mathrm{GeV}$, and the direction of the missing momentum to be in the visible detector region, $\left|\cos \theta_{p_{\text {miss }}}\right|<0.8$.

- Since a large background comes from $W^{+} W^{-}$production, the invariant mass of the lepton and the missing momentum, $m_{l p_{\text {miss }}}$ tends to increase near the $W$ mass. Thus by requiring $m_{l p_{\text {miss }}}>150 \mathrm{GeV}$, that background is reduced.

After these cuts, the remaining background is very small, but $39 \%$ of the signal is retained. With an overall systematic acceptance of $90 \%$ and a total luminosity of $250 \mathrm{fb}^{-1}$ the statistical 
error for the cross-section measurements is

$$
\delta \sigma_{\mathrm{RL}}\left[\chi_{1}^{+} \tilde{\chi}_{1}^{-}\right]=0.6 \%, \quad \delta \sigma_{\mathrm{LR}}\left[\chi_{1}^{+} \tilde{\chi}_{1}^{-}\right]=1.7 \%
$$

where RL and LR stand for the polarization combinations $P\left(e^{+}\right) / P\left(e^{-}\right)=+50 \% /-80 \%$ and $-50 \% /+80 \%$, respectively.

The $\tilde{\chi}_{1}^{ \pm}$and $\tilde{\chi}_{1}^{0}$ masses can be constrained from distributions of the decay products. The summed energy of the two jets from the decay $\tilde{\chi}_{1}^{ \pm} \rightarrow W^{ \pm} \tilde{\chi}_{1}^{0} \rightarrow q \bar{q}^{\prime} \tilde{\chi}_{1}^{0}$ have a well-defined upper endpoint at

$$
E_{\mathrm{jj}, \max }=\frac{\sqrt{s}}{4}\left(1-\frac{m_{\tilde{\chi}_{1}^{0}}^{2}}{m_{\tilde{\chi}_{1}^{ \pm}}^{2}}\right)\left(1+\sqrt{1-4 m_{\tilde{\chi}_{1}^{ \pm}}^{2} / s}\right) .
$$

Moreover, the invariant mass of the same two jets is bounded from above by

$$
m_{\mathrm{jj}, \max }=m_{\tilde{\chi}_{1}^{ \pm}}-m_{\tilde{\chi}_{1}^{0}}
$$

Simple fits to the upper tails of the energy and invariant mass distributions give

$$
E_{\mathrm{jj}, \max }=(120.1 \pm 0.6 \pm 0.5) \mathrm{GeV}, \quad m_{\mathrm{jj}, \max }=(51.8 \pm 0.14 \pm 0.2) \mathrm{GeV}
$$

compared to the model input values $E_{\mathrm{jj}, \max }=120.2 \mathrm{GeV}$ and $m_{\tilde{\chi}_{1}^{ \pm}}-m_{\tilde{\chi}_{1}^{0}}=51.7 \mathrm{GeV}$. Here the first error is the statistical uncertainty of the fit, whereas the second error accounts for the $0.4 \%$ jet energy scale uncertainty. These measurement translate into the following results for the masses:

$$
m_{\tilde{\chi}_{1}^{ \pm}}=(159.4 \pm 1.0) \mathrm{GeV}, \quad m_{\tilde{\chi}_{1}^{0}}=(107.7 \pm 0.9) \mathrm{GeV},
$$

with a large correlation between the two masses. With an independent determination of one of the masses from a different observable, both mass determinations can be improved substantially.

As before, a threshold scan of the chargino cross-section yields a much more precise determination of the chargino mass. Using five scan points with $10 \mathrm{fb}^{-1}$ luminosity each and the same selection cuts as above, a fit to the threshold excitation curve gives

$$
m_{\tilde{\chi}_{1}^{ \pm}}=\frac{1}{2}[\sqrt{s}]_{\mathrm{thr}}=(159.38 \pm 0.04 \pm 0.04) \mathrm{GeV}
$$

where the first error is statistical and the second is the systematic error from the beam energy calibration. Combining eqs. (22) and (24), the masses are obtained as follows,

$$
m_{\tilde{\chi}_{1}^{ \pm}}=(159.38 \pm 0.06) \mathrm{GeV}, \quad m_{\tilde{\chi}_{1}^{0}}=(107.7 \pm 0.21) .
$$




\section{Neutralinos $\tilde{\chi}_{2}^{0}$ and $\tilde{\chi}_{3}^{0}$}

As for the baryogenesis scenario, only the neutralino production process $\tilde{\chi}_{2}^{0} \tilde{\chi}_{3}^{0}$ yields a good signal-to-background ratio. This process has a sizeable cross-section, $\sigma\left[e^{+} e^{-} \rightarrow \tilde{\chi}_{2}^{0} \tilde{\chi}_{3}^{0}\right]=$ $107 \mathrm{fb}$ for unpolarized beams. Using right-handed polarization for the $e^{-}$and left-handed polarization for the $e^{+}$beam, $P\left(e^{+}\right) / P\left(e^{-}\right)=-50 \% /+80 \%$, the signal is enhanced to $129 \mathrm{fb}$, while all Standard Model backgrounds are reduced for this beam polarization combination.

Both the $\tilde{\chi}_{2}^{0}$ and $\tilde{\chi}_{3}^{0}$ almost always decay through a virtual $Z$ boson to a pair of leptons or quarks and the lightest neutralino. As a consequence, the decay products of the two neutralinos cannot be distinguished by kinematical constraints. Therefore, here the semileptonic decay channel is investigated, with one $Z$ decaying leptonically, and the other $Z$ decaying hadronically. Thus the final state is characterized by two charged leptons, two jets and missing energy. As for the baryogenesis case, the relevant Standard Model backgrounds arise from processes with two and three vector bosons and from $t \bar{t}$ production.

The selection cuts to extract the signal from the background are very similar to the baryogenesis case:

- Each event must contain two hadronic jets and two isolated lepton and $p_{\mathrm{t}}^{\text {tot }}>12 \mathrm{GeV}$.

- The invariant mass of either the two jets or the two leptons has to be smaller than $m_{\mathrm{Z}}$, $m_{\mathrm{Z}}-m_{j j}>10 \mathrm{GeV}$ and $m_{\mathrm{Z}}-m_{l l}>10 \mathrm{GeV}$.

- As in section 3.2 it is required that $\left|\cos \theta_{p_{\text {tot }}}\right|<0.9$ and $\not E>100 \mathrm{GeV}$ and a b-quark veto again $t \bar{t}$ background is applied.

A resulting signal efficiency of $47 \%$ is obtained. With a total luminosity of $250 \mathrm{fb}^{-1}$ for the polarization combination $P\left(e^{+}\right) / P\left(e^{-}\right)=-50 \% /+80 \%$, the statistical error for the crosssection measurement is

$$
\delta \sigma_{\mathrm{RL}}\left[\tilde{\chi}_{2}^{0} \tilde{\chi}_{3}^{0}\right]=2.7 \% .
$$

For measurements of kinematic distributions, it is useful to restrict oneself to the two leptons in the final state only, which can be measured more cleanly and precisely than jets. The summed energy of the lepton pair has a distinct upper endpoint, depending from which of the two neutralinos they originate,

$$
\begin{aligned}
& E_{\mathrm{ll}, \max , 2}=\frac{m_{\tilde{\chi}_{2}^{0}}^{2}-m_{\tilde{\chi}_{3}^{0}}^{2}-2 m_{\tilde{\chi}_{1}^{0}}^{2} \sqrt{s}+s}{2 \sqrt{s}}, \\
& E_{\mathrm{ll}, \max , 3}=\frac{m_{\tilde{\chi}_{3}^{0}}^{2}-m_{\tilde{\chi}_{2}^{0}}^{2}-2 m_{\tilde{\chi}_{1}^{0}}^{2} \sqrt{s}+s}{2 \sqrt{s}} .
\end{aligned}
$$

In addition, the invariant mass spectrum of the same two leptons has an endpoint at the mass difference between the intermediate and the final state neutralino,

$$
m_{11, \max , 2}=m_{\tilde{\chi}_{2}^{0}}-m_{\tilde{\chi}_{1}^{0}}, \quad m_{11, \max , 3}=m_{\tilde{\chi}_{3}^{0}}-m_{\tilde{\chi}_{1}^{0}}
$$

The simulated distributions after cuts and fits to the endpoints are summarized in Fig. 囵, For 
(a)

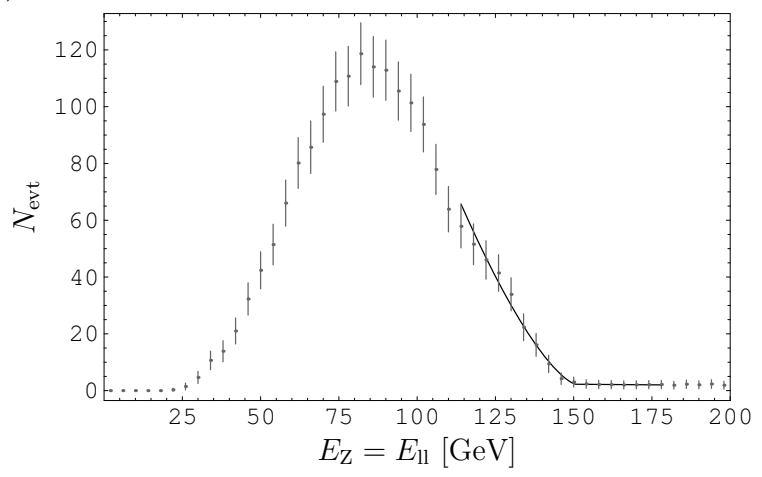

(b)

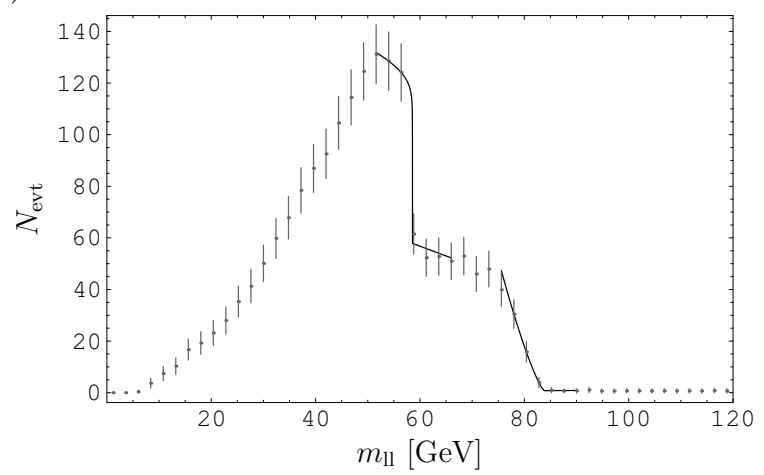

Figure 5: (a) Energy distribution for lepton pair in $\tilde{\chi}_{2,3}^{0} \rightarrow Z \tilde{\chi}_{1}^{0}$ decays, (b) invariant mass of the latter, including simple fitting curves.

the masses in Tab. 4, the upper endpoint of the $E_{\mathrm{ll}, 2}$ spectrum is smaller than the maximum of the $E_{\mathrm{ll}, 3}$ spectrum originating from the $\tilde{\chi}_{3}^{0}$. As a result, the endpoint $E_{\mathrm{ll}, \mathrm{max}, 2}$ gets diluted in the continuum of the $E_{11,3}$ distribution. Therefore, here only the endpoint $E_{11, \max , 3}$ is fitted, but no numerical value for $E_{\mathrm{ll}, \mathrm{max}, 2}$ is obtained. The $m_{\mathrm{ll}}$ shows two characteristic edges corresponding to the contribution from the $\tilde{\chi}_{2}^{0}$ and the $\tilde{\chi}_{3}^{0}$.

Combining fits to these three endpoints, and including a lepton energy scale error of $0.1 \%$, one obtains

$$
\delta m_{\tilde{\chi}_{1}^{0}}=2.9 \mathrm{GeV}, \quad \delta m_{\tilde{\chi}_{2}^{0}}={ }_{-3.1}^{+2.6} \mathrm{GeV}, \quad \delta m_{\tilde{\chi}_{3}^{0}}={ }_{-4.0}^{+4.8} \mathrm{GeV} .
$$

By feeding in the more precise determination of the lightest neutralino mass in eq. (25), these numbers are improved to

$$
\delta m_{\tilde{\chi}_{2}^{0}}={ }_{-2.0}^{+0.3} \mathrm{GeV}, \quad \delta m_{\tilde{\chi}_{3}^{0}}={ }_{-2.3}^{+2.6} \mathrm{GeV} .
$$

From a fit to the cross-sections, not only constraints on the chargino and neutralino parameters are obtained, but also indirect bounds are derived for the masses of the heavy sleptons appearing in the t-channel production contribution (see also Ref. [30]).

The result of the combined fit, taking into account chargino and neutralino cross-section and mass measurements, is

$$
\begin{array}{rlrl}
M_{1} & =\left(123.1_{-0.3}^{+0.4}\right) \mathrm{GeV}, & \tan \beta & =10_{-1.4}^{+0.8} \\
M_{2} & =\left(237.6_{-1.1}^{+0.7}\right) \mathrm{GeV}, & \\
|\mu| & =\left(178.6_{-0.5}^{+0.5}\right) \mathrm{GeV}, & 2.6 \mathrm{TeV}<m_{\tilde{\nu}_{\mathrm{e}}}<4.8 \mathrm{TeV} \\
\left|\phi_{\mu}\right| & <0.6, & m_{\tilde{e}_{\mathrm{R}}}>1.2 \mathrm{TeV}
\end{array}
$$

where the errors indicate one standard deviation $(1 \sigma)$ uncertainties. The precision for the underlying MSSM parameters is comparable to the findings of Ref. [30], although this comparison is precarious since the focus-point scenario studied there is different from the point LCC2. The underlying scenario LCC2 does not contain any CP-violating phases, but nevertheless a non-zero phase of the $\mu$ parameter was allowed in the fit. It turns out that the mass and cross-section observables give only a rather poor constraint on $\phi_{\mu}$. 


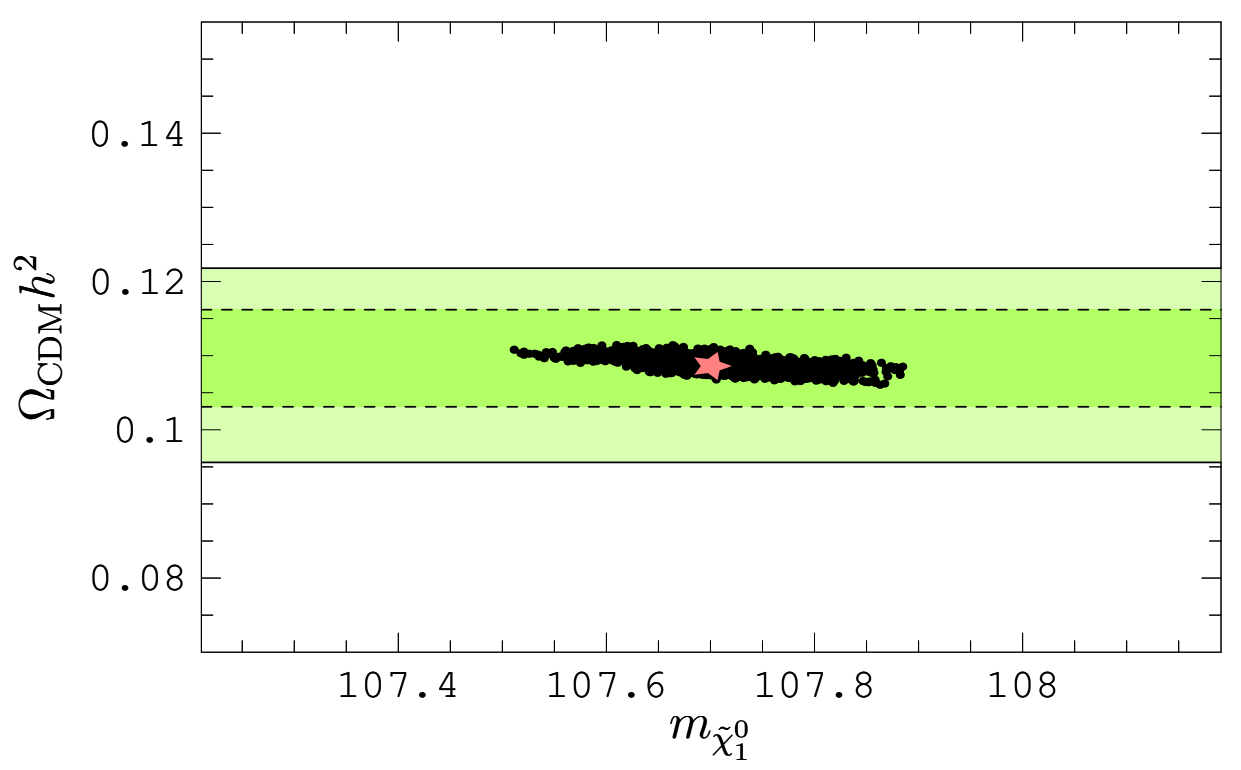

Figure 6: Computation of dark matter relic abundance $\Omega_{\mathrm{CDM}} h^{2}$ for the scenario LCC2, as a function of the lightest neutralino mass $m_{\tilde{\chi}_{1}^{0}}$. The black dots are the $1 \sigma$ allowed region. The original scenario used as input is indicated by the red (light gray) star. The horizontal shaded bands show the $1 \sigma$ and $2 \sigma$ constraints on the relic density measured by WMAP and SDSS.

\subsection{Cosmological implications: dark matter}

In focus-point supersymmetry, the annihilation cross-section is driven by a sizeable higgsino component of the lightest neutralino. As a result, the neutralinos can annihilate efficiently into electroweak gauge bosons. In order to scrutinize this scenario, the neutralino properties need to be determined with high precision. For the sfermions, only indirect bounds can be obtained, but this is sufficient to establish the fact that they effectively decouple from the annihilation cross-section.

The relic dark matter density is computed with DARKSUSY 4.1 [44]. Including the expected precision for the hypothetical neutralino and chargino measurements discussed in the previous section, see eqs. (19), (25), (26), (31), the accuracy for the dark matter determination is obtained from a $\chi^{2}$ scan. It is assumed that a limit of $m_{\tilde{q}}>1000 \mathrm{GeV}$ for the squark masses can be set from non-observation of these particles at the LHC. While in many scenarios it is possible to explore much larger squark masses, a bound of $1000 \mathrm{GeV}$ is sufficient to guarantee that the effect of the squarks on the dark matter annihilation is negligible.

Fig. 6] depicts the result of the scan for the scenario LCC2. The scattered black dots indicate the region allowed by the collider experimental uncertainty, as a function of $m_{\tilde{\chi}_{1}^{0}}$. As pointed out above, although the $\mathrm{LCC} 2$ scenario is $\mathrm{CP}$-conserving, a CP-violating phase for $\mu$ was allowed in the fit to the mass and cross-section measurements. However it was checked that when constraining $\phi_{\mu}$ to zero in the fit, the results remain essentially unchanged. The 
reason for this is that the annihilation cross-section only mildly depends on the CP-phase, but much more strongly on the neutralino mass parameters, which are directly measured. The precision of the predicted relic density $\Omega_{\mathrm{CDM}} h^{2}$ is a very remarkable $2.5 \%$ at $1 \sigma$ level.

The computed relic density depends sensitively on the lightest neutralino mass $m_{\tilde{\chi}_{1}^{0}}$. Compared to Refs. $[27,28]$, the neutralino mass determination is improved in this analysis by the inclusion of the chargino threshold scan. As a result, the dark matter computation from the hypothetical collider measurements is also more accurate, with $\delta\left[\Omega_{\mathrm{CDM}} h^{2}\right] \sim 2.5 \%$ instead of $\delta\left[\Omega_{\mathrm{CDM}} h^{2}\right] \sim 8 \%$ in Ref. [28].

\section{Impact of positron polarization on ILC results}

Throughout the previous sections, is was assumed that both the $e^{-}$and the $e^{+}$beams at the ILC can be polarized, with polarization degrees of $\left|P\left(e^{+}\right)\right|=50 \%$ and $\left|P\left(e^{-}\right)\right|=80 \%$. However, since the realization of positron polarization poses a serious challenge for the accelerator design, it is interesting to investigate how much the results presented above depend on it. As an extreme case, in this section the situation of zero positron polarization is studied, while as before $80 \%$ polarization is assumed for the electron beam, $\left|P\left(e^{+}\right)\right| /\left|P\left(e^{-}\right)\right|=$ $0 \% / 80 \%$. The availability of $e^{-}$polarization ensures that all observables that are considered in the previous sections for the ILC still exist in this case. In particular, supersymmetric production cross-sections can be measured for two different polarization values, $P\left(e^{-}\right)=$ $\pm 80 \%$. However, a loss of accuracy can result from the absence of positron polarization.

In the baryogenesis scenario BGEN, the uncertainty of the determination of the electroweak phase transition strength and of the dark matter density is dominated by the error in the stop mass. The stop mass can be extracted from measuring the stop production cross-section for two different beam polarization combinations. Including $50 \%$ positron polarization [9], an error of $\delta m_{\tilde{t}_{1}}=1.2 \mathrm{GeV}$ is found with this method, while without positron polarization, the error is about $20 \%$ larger, $\delta m_{\tilde{t}_{1}}=1.4 \mathrm{GeV}$. Similarly, the accuracy for the chargino and neutralino parameters $M_{1}, M_{2}$ and $\mu$ is reduced by roughly $20 \%$ with respect to the values in eq. (13) when positron polarization is absent, but these parameters have a smaller impact on cosmological quantities. As a result of the larger stop mass error, a bigger uncertainty for the prediction of the dark matter density is obtained, $\Omega_{\mathrm{CDM}} h^{2}=0.109_{-0.032}^{+0.042}$, compared to ${ }_{-0.027}^{+0.030}$ with $50 \% e^{+}$polarization. As mentioned at the end of section 3.3 , the stop mass can also be determined more precisely from a second cross-section measurement near the stop pair threshold. This method is less sensitive to the beam polarization, and with zero $e^{+}$ polarization the errors are only slightly larger: $\delta m_{\tilde{t}_{1}}=0.32 \mathrm{GeV}$ and $\Omega_{\mathrm{CDM}} h^{2}=0.109_{-0.012}^{+0.015}$, instead of $\delta m_{\tilde{t}_{1}}=0.30 \mathrm{GeV}$ and $\Omega_{\mathrm{CDM}} h^{2}=0.109_{-0.010}^{+0.013}$.

For the focus-point scenario LCC2, the low-energy phenomenology is governed by the chargino and neutralino states. With $0 \%$ instead of $50 \%$ position polarization, the uncertainty of the parameters in eq. (32) is larger by roughly $20 \%$ to $30 \%$. The upper bound that can be extracted for the sneutrino mass from the chargino cross-section measurements gets much weaker, $m_{\tilde{\nu}_{\mathrm{e}}}<13 \mathrm{TeV}$, since the derivation of this parameters is very delicate and requires high precision. The larger errors in the chargino and neutralino parameters also 
translate into a larger error on the predicted dark matter density of $3.1 \%$, compared to $2.5 \%$ with $50 \%$ position polarization. Nevertheless, the possibility to compute the dark matter density with about $3 \%$ precision from collider data is still a very impressive result.

\section{Conclusions}

In various theoretical supersymmetry frameworks, the mass of most or all scalar fermions is constrained to be very large, of the order of at least a few TeV. Typical examples are electroweak baryogenesis in the MSSM, focus-point supersymmetry and split supersymmetry. Focusing on two benchmark points, one electroweak baryogenesis scenario and one focuspoint scenario, it was studied how such a supersymmetry scenario can be explored at future colliders. It was found that the LHC can make discoveries of several superpartner particles in the focus-point case, while in the baryogenesis scenario a new physics signal is likely to be observable at LHC, but the identification of the contributing superpartner particles is very challenging. In both scenarios, the ILC can perform precision measurements to determine the properties of these particles and set bounds on other unobserved states.

The analysis was performed including realistic signal and background computations and simple evaluations of statistical and systematic experimental errors for mass and cross-section measurements. The study was based on the full MSSM, i.e. without assuming a specific mechanism or pattern for supersymmetry breaking parameters.

Using these results, the cosmological implications for electroweak baryogenesis with light stops and stop-neutralino co-annihilation were investigated. It turns out that the collider data helps to elucidate the strength of the electroweak phase transition, while the manifestation of the CP-violating source responsible for the baryon asymmetry remains unconstrained. Furthermore, the collider measurements can be used to compute the relic dark matter density. By determining the stop and lightest neutralino masses, the stop-neutralino co-annihilation process can be strongly constrained and the dark matter density predicted with a precision of the same order as current astrophysical results. Refinements in the determination of the stop mass can improve this result significantly.

In the focus-point scenario, it was found that very precise measurements of the accessible neutralino and chargino states can be performed at the ILC, combining mass measurements from distributions and threshold scans together with cross-section measurements. This allows to set constraints on the slepton mass scale and to compute very accurate predictions for the relic density at the per-cent level. Similar conclusions also apply for split supersymmetry if the lightest chargino and neutralino particles have masses of a few $100 \mathrm{GeV}$.

In both scenarios, the dependence on positron beam polarization was investigated. Assuming $80 \% e^{-}$polarization, and comparing the case of $50 \% e^{+}$polarization to zero $e^{+}$ polarization, it is found that all elements of the analysis can be performed similarly, but roughly $20-30 \%$ precision in the relevant supersymmetry parameters and derived cosmological quantities is lost without $e^{+}$polarization.

The present work has been performed using tree-level formulae and cross-sections for the ILC analysis and the computation of the dark matter annihilation rate. For the expected 
experimental precision, however, radiative corrections are important and introduce a dependence on other supersymmetry parameters, e.g. sfermion masses and mixing outside of the stop sector. Furthermore, no CP-violating phase for the gaugino parameter $M_{1}$ was included in this analysis, which might have interesting effects in the neutralino sector. These issues will be studied in future work.

The present study shows that, even in the challenging case of heavy supersymmetric scalars, precise cross-relations between collider physics and cosmological processes can be established in order to elucidate some of the main unresolved questions in our understanding of the universe.

\section{Appendix: MSSM case study scenarios}

\section{BGEN: Baryogenesis scenario}

The numerical analysis is based on a typical MSSM parameter point characterized by the following weak scale values:

$$
\begin{array}{rlrl}
m_{\tilde{\mathrm{U}}_{3}}^{2} & =-99^{2} \mathrm{GeV}^{2}, & M_{1} & =118.8 \mathrm{GeV}, \\
m_{\tilde{\mathrm{Q}}_{3}} & =4330 \mathrm{GeV}, & M_{2} & =225 \mathrm{GeV}, \\
A_{t} & =-1100 \mathrm{GeV}, \quad|\mu|=225 \mathrm{GeV}, \\
\phi_{\mu} & =0.2, \\
m_{\tilde{\mathrm{Q}}, \tilde{\mathrm{U}}, \tilde{\mathrm{D}}, \tilde{\mathrm{L}}, \tilde{\mathrm{R}}_{1,2}} & =10 \mathrm{TeV}, \quad & \tan \beta & =5, \\
m_{\mathrm{A}^{0}} & =800 \mathrm{GeV} .
\end{array}
$$

Due to constraints from large one-loop sfermion-neutralino and sfermion-chargino effects to the electric dipole moments of the electron and neutron, the sleptons and squarks of the first two generations are chosen to be heavy. The masses of the sbottoms and staus are not specified since they have no relevance for the baryogenesis scenario.

The chosen parameters are compatible with a strongly first order electroweak phase transition for electroweak baryogenesis, $v\left(T_{\mathrm{c}}\right) / T_{\mathrm{c}} \gtrsim 1[5,7]$, generate a sufficiently large baryon asymmetry, $\eta \sim 0.6 \times 10^{-10}$, and yield a value for the dark matter relic abundance $^{2}$ of $\Omega_{\mathrm{CDM}} h^{2}=0.109$, well within the WMAP bounds. Note that the stop-neutralino co-annihilation mechanism is effective for the evolution of the dark matter density in this scenario. Furthermore, the stop parameters are chosen such that the mass of the lightest Higgs boson is $m_{\mathrm{h}^{0}}=117.3 \mathrm{GeV}$, to satisfy the bound from direct searches at LEP $m_{\mathrm{h}^{0}} \gtrsim 114.4 \mathrm{GeV}[24]$. It was checked that the minimum of the scalar potential is color conserving [7]. At tree-level the following masses are obtained for the relevant supersymmetric particles:

$$
\begin{aligned}
& m_{\tilde{t}_{1}}=122.5 \mathrm{GeV}, \quad m_{\tilde{\chi}_{1}^{0}}=106.6 \mathrm{GeV}, \quad m_{\tilde{\chi}_{3}^{0}}=231.2 \mathrm{GeV}, \quad m_{\tilde{\chi}_{1}^{ \pm}}=162.7 \mathrm{GeV}, \\
& m_{\tilde{t}_{2}}=4333 \mathrm{GeV}, \quad m_{\tilde{\chi}_{2}^{0}}=170.8 \mathrm{GeV}, \quad m_{\tilde{\chi}_{4}^{0}}=297.7 \mathrm{GeV}, \quad m_{\tilde{\chi}_{2}^{ \pm}}=296.2 \mathrm{GeV},
\end{aligned}
$$

$\cos \theta_{\tilde{t}}=0.010$.

\footnotetext{
${ }^{2}$ The relic dark matter density has been computed with the code used in Ref. [22].
} 


\section{LCC2: Focus-point scenario}

The point LCC2 is chosen as a point with sizeable gaugino-Higgsino mixing, allowing large neutralino annihilation cross-sections into vector bosons. It was studied previously in Refs. [27, 28]. The scenario is defined by mSUGRA parameters at the unification scale,

$$
m_{0}=3280 \mathrm{GeV}, \quad M_{1 / 2}=300 \mathrm{GeV}, \quad A_{0}=0, \quad \operatorname{sign}(\mu)=+, \quad \tan \beta=10 .
$$

Since the evolution of parameters in the focus-point region sensitively depends on the top quark mass $m_{\mathrm{t}}$, it is fixed to the value $m_{\mathrm{t}}=175 \mathrm{GeV}$. With IsAJET 7.69 [45], the weak scale soft breaking parameters are obtained as follows:

$$
\begin{aligned}
m_{\tilde{\mathrm{U}}_{3}} & =1969 \mathrm{GeV}, \quad M_{1}=123.1 \mathrm{GeV}, \\
m_{\tilde{\mathrm{Q}}_{3}} & =2710 \mathrm{GeV}, \quad M_{2}=237.6 \mathrm{GeV}, \\
m_{\tilde{\mathrm{D}}_{3}} & =3240 \mathrm{GeV}, \quad|\mu|=178.6 \mathrm{GeV}, \\
m_{\tilde{\mathrm{L}}_{3}} & =3268 \mathrm{GeV}, \quad \tan \beta=10, \\
m_{\tilde{\mathrm{R}}_{3}} & =3252 \mathrm{GeV}, \quad m_{\mathrm{A}^{0}}=3242 \mathrm{GeV}, \\
m_{\tilde{\mathrm{Q}}, \tilde{\mathrm{U}}, \tilde{\mathrm{D}}, \tilde{\mathrm{L}}, \tilde{\mathrm{R}}_{1,2}} & \sim 3300 \mathrm{GeV} .
\end{aligned}
$$

For the relevant supersymmetric particle masses, IsAJET 7.69 gives

$$
\begin{array}{lll}
m_{\tilde{\chi}_{1}^{0}}=107.7 \mathrm{GeV}, & m_{\tilde{\chi}_{3}^{0}}=190.0 \mathrm{GeV}, & m_{\tilde{\chi}_{1}^{ \pm}}=159.4 \mathrm{GeV}, \quad m_{\tilde{g}}=850 \mathrm{GeV}, \\
m_{\tilde{\chi}_{2}^{0}}=166.3 \mathrm{GeV}, & m_{\tilde{\chi}_{4}^{0}}=294.4 \mathrm{GeV}, & m_{\tilde{\chi}_{2}^{ \pm}}=286.8 \mathrm{GeV} .
\end{array}
$$

Following the procedure in Ref. [28], the relic density is computed with DARKSUSY 4.1 [44] to $\Omega_{\mathrm{CDM}} h^{2}=0.109$.

\section{Acknowledgments}

The authors are grateful to C. Balázs, E. Baltz, O. Kittel, S. Kraml and T. Plehn for useful discussions. This work was supported by the Schweizer Nationalfonds and by Fermilab, operated by Universities Research Association Inc. under contract no. DE-AC02-76CH03000 with the DOE. A.F. is grateful for hospitality at Fermilab, where part of this work was completed.

\section{References}

[1] D. N. Spergel et al. [WMAP Collaboration], astro-ph/0603449.

[2] M. Tegmark et al. [SDSS Collaboration], Astrophys. J. 606, 702 (2004).

[3] A. I. Bochkarev and M. E. Shaposhnikov, Mod. Phys. Lett. A 2, 417 (1987). 
[4] G. R. Farrar and M. E. Shaposhnikov, Phys. Rev. Lett. 70, 2833 (1993) [Erratum-ibid. 71, $210(1993)]$;

G. R. Farrar and M. E. Shaposhnikov, Phys. Rev. D 50, 774 (1994);

M. B. Gavela, P. Hernandez, J. Orloff, O. Pène and C. Quimbay, Nucl. Phys. B 430, 382 (1994).

[5] M. Carena, M. Quirós and C. E. M. Wagner, Phys. Lett. B 380, 81 (1996).

[6] M. Laine, Nucl. Phys. B 481, 43 (1996) [Erratum-ibid. B 548, 637 (1999)];

M. Losada, Phys. Rev. D 56, 2893 (1997);

G. R. Farrar and M. Losada, Phys. Lett. B 406, 60 (1997);

B. de Carlos and J. R. Espinosa, Nucl. Phys. B 503, 24 (1997);

D. Bodeker, P. John, M. Laine and M. G. Schmidt, Nucl. Phys. B 497, 387 (1997).

[7] M. Carena, M. Quirós and C. E. M. Wagner, Nucl. Phys. B 524, 3 (1998).

[8] M. Laine and K. Rummukainen, Nucl. Phys. B 535, 423 (1998);

M. Losada, Nucl. Phys. B 537, 3 (1999);

M. Losada, Nucl. Phys. B 569, 125 (2000);

M. Laine and M. Losada, Nucl. Phys. B 582, 277 (2000);

M. Laine and K. Rummukainen, Nucl. Phys. B 597, 23 (2001).

[9] M. Carena, A. Finch, A. Freitas, C. Milsténe, H. Nowak and A. Sopczak, Phys. Rev. D 72, 115008 (2005).

[10] T. Ibrahim and P. Nath, Phys. Rev. D 58, 111301 (1998) [Erratum-ibid. D 60, 099902 (1999)].

[11] S. Abel, S. Khalil and O. Lebedev, Nucl. Phys. B 606, 151 (2001).

[12] C. Balázs, M. Carena and C. E. M. Wagner, Phys. Rev. D 70, 015007 (2004).

[13] R. Demina, J. D. Lykken, K. T. Matchev and A. Nomerotski, Phys. Rev. D 62, 035011 (2000).

[14] S. Kraml and A. R. Raklev, Phys. Rev. D 73, 075002 (2006).

[15] A. Finch, H. Nowak and A. Sopczak, in Proc. of International Workshop on Linear Colliders (LCWS 2002), Jeju Island, Korea (26-30 Aug 2002) |hep-ph/0211140|;

A. Bartl, S. Hesselbach, K. Hidaka, T. Kernreiter and W. Porod, hep-ph/0306281.

[16] A. Finch, A. Sopczak and H. Nowak, contributed paper EPS370, International Europhysics Conference on High-Energy Physics (HEP 2003), Aachen, Germany, 17-23 Jul 2003 [LC Note LC-PHSM-2003-075].

[17] B. C. Regan, E. D. Commins, C. J. Schmidt and D. DeMille, Phys. Rev. Lett. 88, 071805 (2002);

C. A. Baker et al., hep-ex/0602020 
[18] J. L. Feng, K. T. Matchev and T. Moroi, Phys. Rev. Lett. 84, 2322 (2000);

J. L. Feng, K. T. Matchev and T. Moroi, Phys. Rev. D 61, 075005 (2000);

K. Agashe, Phys. Rev. D 61, 115006 (2000).

[19] G. F. Giudice and A. Romanino, Nucl. Phys. B 699, 65 (2004) [Erratum-ibid. B 706, $65(2005)]$.

[20] M. Carena, M. Quirós, M. Seco and C. E. M. Wagner, Nucl. Phys. B 650, 24 (2003).

[21] M. Carena, M. Quirós, A. Riotto, I. Vilja and C. E. M. Wagner, Nucl. Phys. B 503, 387 (1997);

J. M. Cline, M. Joyce and K. Kainulainen, JHEP 0007, 018 (2000);

M. Carena, J. M. Moreno, M. Quirós, M. Seco and C. E. M. Wagner, Nucl. Phys. B 599, 158 (2001).

[22] C. Balázs, M. Carena, A. Menon, D. E. Morrissey and C. E. M. Wagner, Phys. Rev. D 71, 075002 (2005).

[23] V. Cirigliano, S. Profumo and M. J. Ramsey-Musolf, hep-ph/0603246.

[24] R. Barate et al. [LEP2 Higgs Working Group, ALEPH, DELPHI, L3 and OPAL Collaborations], Phys. Lett. B 565, 61 (2003);

LEP2 Higgs Working Group, ALEPH, DELPHI, L3 and OPAL experiments, note LHWG-Note-2004-01. [http://lephiggs . web . cern .ch/LEPHIGGS/papers/].

[25] A. Pilaftsis and C. E. M. Wagner, Nucl. Phys. B 553, 3 (1999);

A. Brignole, G. Degrassi, P. Slavich and F. Zwirner, Nucl. Phys. B 643, 79 (2002);

J. S. Lee, A. Pilaftsis, M. Carena, S. Y. Choi, M. Drees, J. R. Ellis and C. E. M. Wagner, Comput. Phys. Commun. 156, 283 (2004);

S. Heinemeyer, W. Hollik and G. Weiglein, hep-ph/0412214.

[26] M. Carena, G. Nardini, M. Quirós and C. E. M. Wagner, in preparation.

[27] R. Gray et al., in Proc. of the International Linear Collider Workshop (LCWS 2005), Stanford, California, 18-22 Mar 2005 hep-ex/0507008.

[28] E. A. Baltz, M. Battaglia, M. E. Peskin and T. Wizansky, hep-ph/0602187.

[29] H. Baer, T. Krupovnickas, S. Profumo and P. Ullio, JHEP 0510, 020 (2005).

[30] K. Desch, J. Kalinowski, G. Moortgat-Pick, K. Rolbiecki and W. J. Stirling, hep-ph/0607104.

[31] K. i. Hikasa and M. Kobayashi, Phys. Rev. D 36, 724 (1987).

[32] A. Freitas, D. J. Miller and P. M. Zerwas, Eur. Phys. J. C 21 (2001) 361;

A. Freitas, A. von Manteuffel and P. M. Zerwas, Eur. Phys. J. C 34 (2004) 487. 
[33] Tesla Technical Design Report, Part IV, eds. T. Behnke, S. Bertolucci, R.D. Heuer and R. Settles, DESY-2001-011D.

[34] S. M. Xella-Hansen, M. Wing, D. J. Jackson, N. de Groot and C. J. S. Damerell, LC Note LC-PHSM-2003-061.

[35] G. Abbiendi et al. [OPAL Collaboration], Eur. Phys. J. C 45, 307 (2006).

[36] J. F. Donoghue, Phys. Rev. D 18, 1632 (1978);

G. Valencia, in Proc. of the Theoretical Advanced Study Institute in Elementary Particle Physics (TASI 94), Boulder, CO, 29 May - 24 Jun 1994 hep-ph/9411441;

Y. Kizukuri and N. Oshimo, in Proc. of Workshop on Perspective of the Electroweak Interaction in $e^{+} e^{-}$Collisions, Ringberg, Germany, 4-8 Apr 1993 hep-ph/9310224.

[37] V. D. Barger, T. Falk, T. Han, J. Jiang, T. Li and T. Plehn, Phys. Rev. D 64, 056007 (2001).

[38] O. Kittel, A. Bartl, H. Fraas and W. Majerotto, Phys. Rev. D 70, 115005 (2004);

A. Bartl, H. Fraas, O. Kittel and W. Majerotto, Eur. Phys. J. C 36, 233 (2004)

[39] M. Acciarri et al. [L3 Collaboration], Phys. Lett. B 439, 225 (1998).

[40] B. C. Allanach, G. Bélanger, F. Boudjema and A. Pukhov, JHEP 0412, 020 (2004);

M. M. Nojiri, G. Polesello and D. R. Tovey, JHEP 0603, 063 (2006).

[41] A. Sopczak, M. Carena, A. Finch, A. Freitas, C. Milsténe and H. Nowak, in Proc. of the 13th International Conference on Supersymmetry and Unification of Fundamental Interactions (SUSY 2005), Durham, England, 18-23 Jul 2005 hep-ph/0605225.

[42] A. Freitas, C. Milsténe, M. Schmitt and A. Sopczak, in preparation.

[43] A. Freitas and P. Z. Skands, hep-ph/0606121.

[44] P. Gondolo, J. Edsjö, P. Ullio, L. Bergström, M. Schelke and E. A. Baltz, JCAP 0407, 008 (2004).

[45] F. E. Paige, S. D. Protopopescu, H. Baer and X. Tata, hep-ph/0312045. 